\title{
The Economics of Clear Advice and Extreme Options*
}

\author{
Dezsö Szalay
}

First version: June 7, 2000

This version: March 28, 2003

\begin{abstract}
This paper is about freedom of choice and rigidity of choice rules as incentive devices. We study the optimal design of an agent's freedom of choice when his information is endogenous and costly to acquire. We show that curtailing the agent's authority over decision-making may be optimal even if the agent's and the principal's ex post objectives coincide. The agent is forced to depart from prior optimal choices and to take a clear stance on a matter. Having the agent choose from extreme options is derived as a second best optimal contract when his information acquisition technology is "success enhancing" and use of contingent monetary compensation infeasible.
\end{abstract}

Key words: discretion, freedom of action, endogenous information

JEL Classification: D82

\footnotetext{
*The paper is a revised version of a Chapter, entitled "Optimal Delegation", of my 2001 doctoral thesis at the University of Mannheim. An earlier version of it circulated under the title "On the One-handed Economist". I owe special thanks to Martin Hellwig for many discussions and comments. Three anonymous referees and Mark Armstrong, the editor, provided excellent comments that greatly improved the paper. I also would like to thank Dirk Bergemann, Jacques Crémer, Mathias Dewatripont, Christian Ewerhart, Sascha Haller, Anke Kessler, Nobuhiro Kiyotaki, Christian Laux, Nicolas Melissas, Benny Moldovanu, John Moore, Georg Nöldeke, Marco Ottaviani, Daniel Probst, and the particpants of various seminars. Financial Support of the Swiss National Science Foundation is gratefully acknowledged. All remaining errors are my own.

Address: Dept. of Economics, HEC-University of Lausanne, BFSH-1, CH-1015 Lausanne, Switzerland; http://www.hec.unil.ch/dszalay; email: dezso.szalay@hec.unil.ch
} 
Winston Churchill: "Should I raise the income tax rate?"

Economist: "Well, on the one hand..., but on the other hand..."

\section{Introduction}

Decision-makers (principals) often lack information and time to gather information they need to make accurate decisions. Therefore they rely on others (agents) to gather information in their stead and provide them with advice, or sometimes even decentralize information acquisition cum decision making - within certain limits. How should such limits be designed? Ex post, when information has been acquired, the principal is concerned with restricting possible abuses by the agent. Ex ante she wants to motivate the agent to acquire information. We develop a stylized model that allows to isolate the role of the ex ante concern and to solve for the optimal freedom of choice relative to this concern.

In our model, a principal needs to decide on a course of action in an uncertain environment. To improve her decision making she hires an agent to gather information at a private cost and to select the course of action on her behalf within the limits she specifies. The principal has carefully screened all potential agents and has hired an agent who has the same preferences over courses of actions she has for any information he may gather. However, screening among (selfish) agents cannot eliminate conflicts of interest ex ante. Even when objectives ex post coincide the agent's information acquisition is optimal relative to his private marginal value of information but suboptimal relative to the social value of information because he neglects the value of information to the principal. Hence, granting unlimited discretion to the agent is efficient ex post but inefficient ex ante. We establish a sequence of results in this environment. First, restricting the agent's freedom of choice can increase his incentive to acquire information. More specifically, if the agent is forced to depart from the act which is optimal relative to his prior information then he finds it relatively more attractive to have precise rather than imprecise information. Second, the benefit arising from the resulting improvement in the quality of information may outweigh the cost arising from the resulting inefficient use of information ex post. Third, prohibiting the agent from selecting the prior optimal act is shown to be the optimal restriction on his choice set.

Our theory explains why it is optimal to force economists to give clear answers: they think harder before they answer. E.g., competition authorities are usually forced to take a clear stance on whether a proposed merger is detrimental to welfare or not. The answer "On the one hand..., 
but..." is not an admissible option. Similarly, jurors and judges have to choose between the extreme options "guilty" and "not guilty". The absence of a compromising choice makes them think harder about whether the accused is indeed guilty or not. The argument applies to situations of decentralized and centralized decision-making alike. In situations of centralized decision-making we argue that the principal should commit to not follow the agent's advice if he recommends the prior optimal act. To be specific, consider corporate decision-making where a division manager is in charge of screening projects relative to the status quo. We argue that expected profits may be higher if headquarters commits to activism and imposes an innovation bias on the firm than if it adopts a more flexible policy because division managers have more of an incentive to screen projects. Finally, consider delegated portfolio-management. According to our theory an investor may benefit from forcing her portfolio-manager to depart from a passive strategy and to take risks. Having to decide whether to go short or long in an asset the manager has more of an incentive to gather information about returns. ${ }^{1}$

The paper belongs to a literature that tries to explain institutions of decision-making as best responses to problems of asymmetric information. Most closely related in terms of analysis are Holmström (1978, 1984), Armstrong (1994), and Aghion and Tirole (1997). Holmström and Armstrong have studied how a principal should optimally delegate a task to an agent who is not trustworthy but has exogenously given, superior information. The optimal form of delegation trades off the gains arising from the use of the agent's information against the losses arising from preference divergence ex post. Aghion and Tirole (1997) discuss the optimal allocation of decision rights when information is endogenous. Delegating formal decision rights to an agent may be optimal even though the latter sometimes abuses the freedom of action this provides because the agent has more of an incentive to acquire information. In contrast to Aghion and Tirole (1997) our theory predicts that less freedom of action may provide more of an incentive to acquire information. The reason is that we take actions as contractible while in Aghion and Tirole (1997) only the unconstrained right to decide is contractible. ${ }^{2}$ Most closely related in terms of results is $\mathrm{Li}(2001)$,

\footnotetext{
${ }^{1}$ The application of our theory to portfolio-management is discussed in more detail in a companion paper (Szalay $(2003))$.

${ }^{2}$ Tirole (1999) develops the "complete contracting" version of the Aghion and Tirole (1997) paper. He discusses the institution of giving authority to the agent with the principal having "gatekeeping counterpower". In this institutional setting the principal is given the right to exclude certain alternatives a priori. However, his point is to show that the concept of authority is not an artefact of modelling the problem in an incomplete contracting framework. To illustrate this idea, he shows that we can find an institution in an incomplete contracting context that implements the same outcome as the optimal complete contract. Giving authority to the agent with the principal
} 
who explains excessive conservatism in committee decision-making as a contractual response to free-rider problems in fact-finding. As in the present paper, ex post inefficient decision-making is used to alleviate the ex ante incentive problem in information acquisition. However, in contrast to $\mathrm{Li}$ we take evidence as nonverifiable. More importantly, Li studies a binary choice problem while we take the number of alternatives taken in equilibrium as endogenous and derive them as a second best contract. Dewatripont and Tirole (1999) study a related decision problem. However, they do not focus on freedom of action as an incentive device but rather explain specialization in information acquisition as a best response to incentive problems in information acquisition. Prendergast (1993) explains why agents are given incentives to second-guess their bosses' opinions. The ability to second-guess is evidence (in the contract theoretic sense) in favor of hard work on information acquisition. In contrast to Prendergast's model our principal does not acquire information and gives the agent incentives to come up with information different from hers. Confirming the principal's prior information is evidence against hard work on information acquisition. Finally, Demski and Sappington (1987), and more recently Crémer et.al. (1998a) and Lewis and Sappington (1997) have studied similar problems of contracting with endogenous information. As the present analysis these papers study problems of moral hazard in information acquisition followed by an adverse selection problem. The most significant novelty relative to these approaches is our focus on freedom of choice as an incentive device. ${ }^{3}$

Before proceeding to the analysis we wish to point to the important modeling choices in the paper. First, we rely on a strong notion of commitment. Once information is acquired there is unanimous agreement on the optimal course of action. Hence, principal and agent have an incentive to renegotiate for an ex post efficient use of information. We assume that no such renegotiation takes place. Therefore, the theory applies to situations in practice where commitment can be taken for granted, e.g., to judicial decision-making, for the law cannot be changed in every trial, to firm policies that are deeply routed in corporate cultures, or to situations where reputation concerns keep the principal from reneging. When commitment can be taken for granted we show that there having gatekeeping counterpower is an example of such an institution. This point is distinct from our question of how to use gatekeeping counterpower optimally.

${ }^{3}$ See Laffont and Martimore (2002) for a discussion of these models. In such problems contracts must simultaneously induce optimal information gathering and truthful revelation of information. Contracts involve additional distortions relative to the case with exogenous information reflecting the marginal value of information. Further papers in this vein are Crémer et.al. (1998b), Crémer and Khalil (1992), Laux (2001), and Kessler (1998). Relatedly, Bergemann and Välimäki (2002) have analyzed the incentives ex post efficient mechanisms provide for information acquisition. 
is value to it arising from the incentive effect it provides. ${ }^{4}$ Second, we focus on the case where the adviser has a partisan objective. That is, we neglect the role of implicit incentives provided by career concerns as analyzed, e.g., by Prendergast and Stole (1996) or Holmström and Ricart-iCosta (1986). ${ }^{5}$ Consequently, our theory applies when agents' concerns for the direct consequences of courses of actions are relatively more important to them than implicit incentives provided by career concerns.

The remainder of this article is organized as follows. Section 2 introduces the model. We first analyze the case of an agent who is infinitely risk averse so that the use of the transfer scheme is mute (section 3). In section 4 we study the case where the agent is risk neutral with respect to money payments. Section 5 discusses extensions of the results in various directions. All proofs are relegated to the appendix.

\section{The model}

We consider an agency problem in which the utility of both the principal and the agent depend on an action, $x$, as well as on a parameter, $\eta$, according to the specification

$$
V(x, \eta)=K-\pi(x, \eta)
$$

and

$$
U(x, \eta, \alpha)=\alpha(K-\pi(x, \eta))
$$

and where $V(\cdot, \cdot)$ is the utility of the principal, $U(\cdot, \cdot, \cdot)$ the utility of the agent, and $\pi(x, \eta)$ is a quadratic loss function. More specifically,

$$
\pi(x, \eta)=\frac{1}{2}(x-\eta)^{2}
$$

The parameter $\alpha$ measures the relative value of information to the agent (see below). We assume that $0<\alpha<\infty$. The parameter $\eta$ is assumed to be the realization of a random variable $\tilde{\eta}$, taking values in an interval $[\underline{\eta}, \bar{\eta}]$. We assume that the distribution of $\tilde{\eta}$ has a density $f(\eta)$ with full

\footnotetext{
${ }^{4}$ Recently, Dessein (2001) has shown that there is value to commitment in the delegation problem arising from purely informational concerns. Dessein shows that the harm arising from loss of information in noisy communication à la Crawford and Sobel (1982) is larger than the harm arising from conflicts of interests under delegation when preference divergence is not too pronounced. De Garidel-Thoron and Ottaviani (2000) extend this comparative analysis of institutions allowing for a varying degree of strategic sophistication on the part of the receiver.

${ }^{5}$ For a simultaneous consideration of cheap talk and reputational concerns and for a more extensive reference to these issues, see Ottaviani and Sorensen (2000).
} 
support. The expected value of $\tilde{\eta}$ is $\mu, \sigma$ is the strictly positive and finite standard deviation of $\tilde{\eta}$. The set of a priori feasible actions, $x$, is $[\underline{\eta}, \bar{\eta}]$. For future reference we denote $W(x, \eta)$ as the joint utility of the principal and the agent, i.e.,

$$
W(x, \eta)=U(x, \eta, \alpha)+V(x, \eta)=(1+\alpha)(K-\pi(x, \eta))
$$

At the time of contracting both agent and principal know $f(\eta)$ but neither of them knows the realization of $\tilde{\eta}$. Between the time the contract is written and the choice of the action $x$ the agent may perform a location experiment on $\eta$. If the agent exerts effort $e$ the experiment is a success with probability $e$ and is unsuccessful with probability $(1-e)$. A successful experiment reveals the realization of $\tilde{\eta}$ to the agent. If the experiment fails, the agent does not acquire additional information. Hence, the agent's effort is success enhancing in the sense of Green and Stokey (1981). ${ }^{6}$ The cost of an experiment that succeeds with probability $e$ is $g(e)$, where $g(e)$ is increasing and convex, and satisfies the INADA conditions ${ }^{7}: g_{e}(e)>0 \forall e>0, g_{e e}(e)>0 \forall e ;\left.\left(g_{e}(e)\right)\right|_{e=0}=0$; $\lim _{e \rightarrow 1} g_{e}(e)=\infty$. The agent's choice of $e$ is not observable to the principal. If the experiment is successful the realization of $\tilde{\eta}$ is the agent's private information. Moreover, the principal does not observe whether the experiment is a success or not so that his information is soft and can be forged.

Before the agent performs the experiment the principal commits to a mechanism. She specifies a message space $M$ so that the agent can send messages $m \in M$ to the principal once he has observed the outcome of the experiment and commits to taking the action $x(m)$ and paying a transfer $t(m, \pi(x(m), \eta))$ to the agent if his message is $m$ and the realized loss is $\pi(x(m), \eta)$. We assume that the principal must commit to this mechanism. In particular, she cannot renege on the choice scheme $x(m)$.

The principal is risk neutral with respect to money income. The agent's utility is additively separable in decision dependent utility, (2), utility from money income, and costs of effort. He is infinitely averse to income risk. He therefore does not respond to monetary incentives and receives a constant payment, $t$, equal to his outside wage, which is normalized to zero. ${ }^{8}$ The assumption of infinite risk aversion will be relaxed in section 4 .

Consider now the nature of the incentive problem. From (1) and (2) it is obvious that there is no conflict of interest with respect to the choice of $x$ ex post. However, ex ante the principal and

\footnotetext{
${ }^{6}$ Alternative location experiments are discussed in section 5 below.

${ }^{7}$ Throughout the paper, subscipts denote derivatives of functions.

${ }^{8}$ This is the standard assumption to justify nonmonetary contracting. We follow Aghion and Tirole (1997) p.6.
} 
the agent disagree on the choice of $e$. To see this, suppose that information is used efficiently $e x$ post so that $x=\eta$ is chosen when information $\eta$ is available and $x=\mu$ is chosen when only prior information is available. The marginal value of information to the agent is equal to the expected incremental utility he obtains when information $\eta$ becomes available, $\frac{\alpha \sigma^{2}}{2}$. The agent exerts effort until the marginal cost of effort, $g_{e}(e)$, equals the private marginal value of information. By contrast, the social value of information includes the value of information to the principal, $\frac{\sigma^{2}}{2}$. Consequently, the agent exerts too little effort from a social perspective if information is used efficiently ex post. The question is then, whether a commitment by the principal to use information inefficiently ex post raises ex ante expected welfare?

\section{Freedom of Choice as an Incentive Device}

By the revelation principle we can think of a contract as specifying a direct, incentive compatible mechanism. The agent communicates his information to the principal and is given incentives to be truthful. Given that the principal cannot use monetary transfers, she never can do better than giving the agent the right to choose an action $x$ out of a closed subset of $[\underline{\eta}, \bar{\eta}] .{ }^{9}$ We let $\boldsymbol{\Gamma}$ denote the set of closed subsets of $[\underline{\eta}, \bar{\eta}]$ with typical element $\Gamma$. From this perspective, the principal's problem is to choose a closed set $\Gamma$ and a constant payment $t$, with the interpretation that the agent is free to choose $x \in \Gamma$. We let $x(\eta)$ denote his choice of act if he has precise information and $x(\phi)$ denote his choice of act if his information is coarse. The principal's problem is ${ }^{10}$ :

$$
\begin{gathered}
\max _{\Gamma \in \Gamma, t} e E V(x(\eta), \tilde{\eta})+(1-e) E V(x(\phi), \tilde{\eta})-t \\
\text { s.t. } \\
x(\eta) \in \arg \max _{x \in \Gamma} U(x, \eta, \alpha) \quad \forall \eta \\
x(\phi) \in \arg \max _{x \in \Gamma} E U(x, \tilde{\eta}, \alpha) \\
E U(x(\eta), \tilde{\eta}, \alpha)-E U(x(\phi), \tilde{\eta}, \alpha)=g_{e}(e) \\
e E U(x(\eta), \tilde{\eta}, \alpha)+(1-e) E U(x(\phi), \tilde{\eta}, \alpha)-g(e)+t \geq 0
\end{gathered}
$$

\footnotetext{
${ }^{9}$ This was first observed by Holmström $(1978,1984)$ and Green and Stokey (1981). We use the result in the spirit of Melumad and Shibano (1991).

${ }^{10}$ Throughout the paper $E$ denotes the expectation operator.
} 
The first term in (5) represents the principal's expected payoff conditional on a successful information acquisition experiment weighted by the probability of success, e. It takes into account (6), i.e., that the agent will choose his most preferred alternative for each realization of $\tilde{\eta}$ subject to the restriction $\Gamma$. The second term is the analogue for the case where the agent chooses $x$ after an unsuccessful experiment. In this case the incentive compatibility condition on the agent's choice of $x$ is (7) . (8) is the incentive compatibility condition for the agent's effort choice. It states that the agent's level of effort equates the private marginal value of information to the marginal cost. (9) states that the agent must be willing to go along with the principal's contract proposal ${ }^{11}$. Since the principal has quasilinear utility and unlimited wealth and the agent is infinitely risk averse, (9) is binding at the optimum and the principal maximizes expected social surplus subject to incentive compatibility of the agent's choices, $x$ and $e$.

Our first result is that it is optimal to have the agent choose any act he likes, with the possible exception of acts that are close to the prior optimal act, $\mu$. Let $\Gamma^{*}$ denote an optimal contract. Then:

Proposition $1 \Gamma^{*} \in \Gamma^{D}$ where

$$
\Gamma^{D}=\{\Gamma \in \Gamma: \Gamma=[\underline{\eta}, \bar{\eta}] \backslash(\mu-d, \mu+d) \text { for all } d \in[0, \min \{\mu-\underline{\eta}, \bar{\eta}-\mu\}]\}
$$

If the principal wants to mitigate the consequences of the agent's underprovision of effort, she must (by (8)) increase the marginal value of information to the agent, $E U(x(\eta), \tilde{\eta}, \alpha)-$ $E U(x(\phi), \tilde{\eta}, \alpha)$. She cannot increase $E U(x(\eta), \tilde{\eta}, \alpha)$ relative to the case where the agent has unlimited discretion, because conditional expected utility conditional on a successful experiment is maximized if information is used efficiently. Therefore, she must reduce the agent's expected utility conditional on experiment failure, i.e., punish him if the experiment fails. Since $E U(x(\phi), \tilde{\eta}, \alpha)$ is decreasing in the Euclidian distance between the agent's restricted preferred act, $x(\phi)$, and his unrestricted preferred act, $\mu$, the principal can reduce $E U(x(\phi), \tilde{\eta}, \alpha)$ by forcing the agent to depart from $\mu$ by $\Delta$, say. The choice sets she can offer to enforce this departure from $\mu$ in an incentive compatible way must contain at least one act with distance $\Delta$ to $\mu$ and must not contain any acts closer to $\mu$ than this act. Contracts in $\boldsymbol{\Gamma}^{D}$ are optimal in this set of incentive compatible contracts because they minimize on the cost of inflicting a given punishment on the agent. This is simply because prohibiting the agent from choosing acts with more than distance $\Delta$ to $\mu$ does

\footnotetext{
${ }^{11}$ For simplicity, the coefficient of proportionality on the money term in the agent's utility is assumed to be equal to one.
} 
not influence the punishment but prohibits him from choosing the ex post efficient act for some realizations of $\eta$ if the experiment succeeds. Consequently, the principal removes exclusively a convex set, containing $\mu$, from the agent's choice set. Finally, an optimal choice set contains the bounds of the set of feasible acts, $\underline{\eta}$ and $\bar{\eta}$. Removing an extreme policy dramatically reduces the sensitivity of $x(\eta)$ with respect to the agent's information and reduces his incentive to acquire information. By consequence, the set of removed actions is also symmetric around $\mu$. Letting $\Delta^{*}$ denote an optimal choice of punishment for experiment failure by the principal and letting $\Gamma^{*}=[\eta, \bar{\eta}] \backslash\left(\mu-\Delta^{*}, \mu+\Delta^{*}\right)$ denote an optimal contract, these arguments show that $\Gamma^{*} \in \boldsymbol{\Gamma}^{D}$.

The principal optimally excludes a convex set from the agent's choice set regardless of the distribution of $\eta$. This result contrasts with Holmström's (1984) analysis of delegation contracts with preference divergence and given information, where convex prohibitions are optimal only if the distribution $F(\cdot)$ is uniform. The difference is due to the simplicity of our agency problem $e x$ post as well as the simplicity of the information acquisition technology. Moreover, in our context the set of optimally prohibited acts is even symmetric, regardless of the distribution $F(\eta)$. This is due to the symmetry of the agent's payoff function. In a more general analysis allowing for asymmetric utility functions, the optimal contract might well be asymmetric. However, as long as the information acquisition technology is success enhancing the prohibited set would still be convex.

By proposition 1, the principal's problem reduces to choosing the bounds, $\mu \pm \Delta$, of a symmetric interval around the mean optimally. Conditional expected losses conditional on the agent's information and conditional on incentive compatibility of his choices under contracts of the optimal structure can be written as

$$
\begin{aligned}
E \pi(x(\eta), \tilde{\eta})= & \frac{1}{2} \int_{\mu}^{\mu+\Delta}(\mu+\Delta-\eta)^{2} d F(\eta)+\frac{1}{2} \int_{\mu-\Delta}^{\mu}(\mu-\Delta-\eta)^{2} d F(\eta) \\
& \text { and } \\
E \pi(x(\phi), \tilde{\eta})= & \frac{1}{2}\left(\sigma^{2}+\Delta^{2}\right)
\end{aligned}
$$

We therefore simplify our notation and let $E \pi(\Delta, \eta):=E \pi(x(\eta), \tilde{\eta})$ and $E \pi(\Delta, \phi):=E \pi(x(\phi), \tilde{\eta})$. The notation for the agent's expected utility, $E U(\cdot, \cdot, \alpha)$, and joint expected utility, $E W(\cdot, \cdot, \alpha)$, is simplified accordingly. From (8), the agent's incentive compatible effort choice is

$$
e(\Delta, \alpha)=g_{e}^{-1}[\alpha(E \pi(\Delta, \phi)-E \pi(\Delta, \eta))]
$$

where $g_{e}^{-1}(\cdot)$ exists because $g(e)$ is strictly convex. We next study how the agent's incentive compatible effort choice depends on the size of the convex prohibition. 
Proposition $2 e(\Delta, \alpha)$ is strictly increasing in $\Delta$.

Since the outcome of the experiment is unobservable to the principal, forcing the agent to depart from the prior optimal choice decreases expected conditional utilities both conditional on failure and on success. However, the reduction of the former level of expected utility is always larger than the reduction of the latter. Conditional on an unsuccessful experiment the agent would like to choose the prior optimal act with probability one and must deviate by $\Delta$ from this act. Conditional on a successful experiment the agent's choice of $x$ is affected only in the event that $\eta \in(\mu-\Delta, \mu+\Delta)$. Moreover, the induced deviation from the agent's unrestricted preferred act is almost surely smaller than $\Delta$. Therefore the inability to choose can be used to provide incentives. ${ }^{12}$

Consider now the optimal choice of $\Delta$. Using (11) one can write the principal's problem, (5) s.t. (6)-(9), as the unconstrained maximization problem

$$
\left.\max _{\Delta} P(\Delta, \alpha)=E W(\Delta, \phi, \alpha)+e(\Delta, \alpha)\right)(E W(\Delta, \tilde{\eta}, \alpha)-E W(\Delta, \phi, \alpha))-g(e(\Delta, \alpha))
$$

The principal's optimal choice of $\Delta$ trades off the benefit arising from increasing the expected precision of the agent's information against the loss arising from using his information inefficiently ex post. Let $\Delta^{*}$ denote an optimal solution to problem (12). For prohibitions of infinitesimal size the benefit may outweigh the cost.

Proposition 3 The principal's problem has a solution. $\Delta^{*}$ is strictly positive if the function $g(\cdot)$ and the parameters $\alpha$ and $\sigma$ satisfy the inequality

$$
\left.\left(\frac{g_{e}(e(\Delta, \alpha))}{g_{e e}(e(\Delta, \alpha))}\right)\right|_{\Delta=0}>\left.(1+\alpha)(1-e(\Delta, \alpha))\right|_{\Delta=0}
$$

where $\left.e(\Delta, \alpha)\right|_{\Delta=0}=g_{e}^{-1}\left(\frac{\alpha \sigma^{2}}{2}\right)$ is the level of effort the agent exerts under full freedom of action.

The principal's payoff function is continuous in $\Delta$ so that a maximizer exists. Moreover, as we show in the appendix, it has a stationary point at $\Delta=0$. Consequently, $\Delta^{*}>0$ if the stationary point at zero satisfies the second order condition for a local minimum, which is precisely what (13) asserts. The benefit of introducing a small restriction around $\mu$ is that the agent exerts a higher level of effort so that the probability of a successful experiment is increased. At the margin this

\footnotetext{
${ }^{12}$ Again, it is interesting to contrast our result with Holmström's. He observed that giving more freedom of action to the agent in the sense of giving him more discretion to choose among extreme options provides the agent with more of an incentive to acquire information (see Holmström (1978) p.98). The same is true for our problem (see Szalay (2000)). However, in contrast to the case with conflicting interests, there is no reason to prohibit the agent from choosing extreme actions in the first place.
} 
benefit accrues only to the principal, because the agent's choice of effort is optimal relative to his private value of information. Her benefit is inversely proportional to the curvature of the cost-ofeffort function evaluated at the level of effort the agent is willing to exert under full freedom of action. The less curved the cost-of-effort function relative to its slope, the better the agent responds to incentives and the higher the benefit. The cost of introducing a restriction is that information is used inefficiently so that ex post payoffs are reduced. An infinitesimal restriction has no (first and second order) effect on $E W(\Delta, \tilde{\eta}, \alpha)$ but an (second order) effect on $E W(\Delta, \phi, \alpha)$. The expected reduction of joint payoff is equal to $\left.(1+\alpha)(1-e(\Delta, \alpha))\right|_{\Delta=0}$. A cost-of-effort function satisfying condition (13) always exists:

Proposition 4 For any positive and bounded $\alpha$ there exists a convex INADA cost function $g(\cdot)$ such that (13) is satisfied. More specifically, let

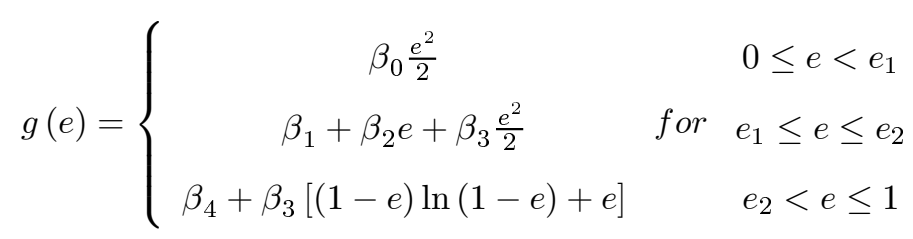

where $e_{1}=\frac{\beta_{2}}{\beta_{0}-\beta_{3}}$, e $e_{2}$ is the positive solution to $\beta_{2}+\beta_{3} e=-\beta_{3} \ln (1-e)$, and $\beta_{1}$ and $\beta_{4}$ are chosen such that $g(e)$ is continuous in e. Then, there exist values of the parameters $\beta_{0}, \beta_{2}$, and $\beta_{3}$, with $\beta_{3}$ small relative to $\beta_{2}$, such that $e_{1}<\left.e(\Delta, \alpha)\right|_{\Delta=0}<e_{2}$ and (13) is satisfied.

For any positive and bounded values of the parameters $\alpha$ and $\sigma$ one may find a cost function which is close enough to linear around the effort level the agent exerts without extra incentives so that (13) is indeed satisfied. More precisely think of $g(\cdot)$ as of a second order polynomial around $\left.e(\Delta, \alpha)\right|_{\Delta=0}$ and let the second order coefficient go to zero for a fixed first order coefficient. Then, the response of the agent to the incentive system goes out of bounds while the cost in terms of the probability of inefficient decision-making ex post remains bounded, because the level of effort is determined primarily by the fixed first order coefficient.

How large should an optimal prohibition be? To answer this question we must impose more structure on our model. More specifically, we shall assume for the remainder of this paper that the cost-of-effort function is a $C^{2}$ function. Until further notice we shall also restrict it to the multiplicative form $g(e)=\beta h(e)$ where $\beta$ is a positive parameter and $h(e)$ a convex INADA function. In this case it is straightforward that the agent's incentive compatible effort choice under a contract of the optimal form satisfies

$$
e\left(\Delta, \frac{\alpha}{\beta}\right)=h_{e}^{-1}\left[\frac{\alpha}{\beta}(E \pi(\Delta, \phi)-E \pi(\Delta, \tilde{\eta}))\right]
$$


Note that the incentive compatible effort level is montone decreasing in $\beta$. Let $\left.e\left(\Delta, \frac{\alpha}{\beta}\right)\right|_{\Delta=0}=$ $h_{e}^{-1}\left(\frac{\alpha \sigma^{2}}{2 \beta}\right)$ denote the effort level the agent exerts under full freedom of action. We next investigate for which values of the parameter $\beta$ the principal's net benefit of introducing small restrictions on the agent's choice set is positive.

Proposition 5 Let $g($ e) take a multiplicative form so that

$$
g(e)=\beta h(e)
$$

where $\beta$ is a strictly positive parameter and $h(e)$ any convex INADA function which is $C^{2}$ and satisfies a) $\lim _{e \rightarrow 1} \frac{h_{e}(e) h_{e e e}(e)}{\left(h_{e e}(e)\right)^{2}}>2+\alpha$ and b) $\frac{h_{e}(e)}{h_{e e}(e)}$ concave in $e$. Then, for any strictly positive and bounded values of the parameters $\alpha$ and $\sigma$, there exists a unique, strictly positive value of $\beta$, $\beta^{\prime}$, such that (13) is satisfied iff $\beta \in\left(0, \beta^{\prime}\right)$.

Conditions a) and b) are satisfied by the following INADA cost-of-effort function:

$$
g(e)=\beta((1-e) \ln (1-e)+e)
$$

Note that the value of the agent's reponsiveness to incentives does not depend directly on $\beta$ for multiplicative cost-of-effort functions but only indirectly through the dependence of the agent's effort choice on $\beta$. In the limit as $\beta$ goes to zero, the agent supplies the socially optimal level of effort - which equals one in the limit - so that the value of increasing his effort is zero. On the other hand the cost of increasing his effort choice are also zero because he has precise information with probability 1 so that he almost surely wants to choose an act different from $\mu$. Hence, the left-hand side and the right-hand side of (13) exactly match and the inequality cannot be satisfied in the limit as $\beta$ goes to zero. If condition a) holds then the cost-of-effort function displays relatively little curvature relative to its slope for high effort levels just below 1. If in addition condition b) holds then the net benefit, defined by the difference between the left-hand and the right-hand side of (13) is concave in $e$. Since the agent's effort and his responsiveness to incentives goes to zero in the limit as $\beta$ goes to infinity the net benefit is negative in this limit. Hence, (13) is satisfied if and only if $\beta$ is small enough.

By direct extension of $(12)$ let $P\left(\Delta, \alpha, \frac{\alpha}{\beta}\right)$ denote the principal's payoff function when $g(e)$ is of the multiplicative form. Let $\Delta^{*}$ denote an optimal solution to the principal's problem. $\Delta^{*}$ satisfies the first order condition $\left.P_{\Delta}\left(\Delta, \alpha, \frac{\alpha}{\beta}\right)\right|_{\Delta_{=\Delta^{*}}}=0$, i.e.,

$$
\left.\left(\left((1+\alpha) e\left(\Delta, \frac{\alpha}{\beta}\right)+\frac{g_{e}\left(e\left(\Delta, \frac{\alpha}{\beta}\right)\right)}{g_{e e}\left(e\left(\Delta, \frac{\alpha}{\beta}\right)\right)}\right)\left(E \pi_{\Delta}(\Delta, \phi)-E \pi_{\Delta}(\Delta, \eta)\right)-(1+\alpha) \Delta\right)\right|_{\Delta_{=\Delta^{*}}}=0
$$


The principal trades off the marginal benefit from punishing the agent, arising from the expected increase in precision of the agent's information, to the marginal cost arising from inefficient use of information. More specifically, the marginal benefit is the sum of the expected marginal increase in the net social gain from a successful relative to an unsuccessful experiment, $e\left(\Delta, \frac{\alpha}{\beta}\right)\left(E W_{\Delta}(\Delta, \eta)-E W_{\Delta}(\Delta, \phi)\right)$, and the marginal increase in the agent's effort choice multiplied by the principal's net gain from a successful experiment relative to an unsuccessful experiment, $\frac{\partial e\left(\Delta, \frac{\alpha}{\beta}\right)}{\partial \Delta} E V_{\Delta}(\Delta, \eta)-E V_{\Delta}(\Delta, \phi)$. Net marginal benefits accruing to the agent are zero, because his optimal effort choice equalizes the marginal benefit from acquiring precise information to the marginal cost. The marginal cost is the welfare loss in case the experiment fails, $E W_{\Delta}(\Delta, \phi)$. Simple manipulations, using (10) and (14) deliver the more tractable formulation of the first order condition (15). Define $\underline{\Delta}$ as the smallest local maximizer of the principal's payoff function. Formally $\underline{\Delta}=\min \left\{\Delta: P_{\Delta}\left(\Delta, \alpha, \frac{\alpha}{\beta}\right)=0\right.$ and $\left.P_{\Delta \Delta}\left(\Delta, \alpha, \frac{\alpha}{\beta}\right)<0\right\}$.

Proposition 6 Suppose that $\lim _{e \rightarrow 1} \frac{h_{e}(e) h_{e e}(e)}{h_{e e}(e)^{2}}>2+\alpha$ and that $\frac{h_{e}(e)}{h_{e e}(e)}$ is concave in e. Then, there exists a unique value of the parameter $\beta, \beta^{\prime \prime}$, satisfying $\beta^{\prime}>\beta^{\prime \prime}>0$ such that $\triangleq$ has the following properties:

i) $\Delta$ tends to zero in the limit as $\beta$ tends to zero. $\Delta$ is increasing in $\beta$ for $\beta \in\left(0, \beta^{\prime \prime}\right]$, decreasing in $\beta$ for $\beta \in\left(\beta^{\prime \prime}, \beta^{\prime}\right]$, and equal to zero for $\beta>\beta^{\prime}$.

ii) If $\beta \leq \beta^{\prime \prime}$ then $\Delta^{*}=\underline{\Delta}$.

By definition, $\underline{\Delta}>0$ iff (13) is satisfied. As $\beta$ is decreased below $\beta^{\prime} \underline{\Delta}$ increases first and then decreases until it attains a value of zero in the limit as $\beta$ goes to zero. This is because the principal's marginal benefit arising from a punishment of given size is a concave function of the agent's effort choice and because the effort choice under contract $\Delta$ is monotonic in $\beta$ even though $\underline{\Delta}$ is nonmonotonic. Economically, as $\beta$ is decreased below $\beta^{\prime}$, the agent reacts better to incentives and the expected cost of using information inefficiently decreases because the level of effort the agent exerts for any given contract increases. The assumption of a concave value of responsiveness implies that the difference between first best level of effort and the level of effort the agent exerts under full freedom of action is decreasing in $\beta$ for $\beta \in\left(0, \beta^{\prime \prime}\right]$. Therefore, the principal has less of an incentive to increase the agent's effort choice and reduces the size of the prohibition if $\beta$ is decreased. In general, $\underline{\Delta}$ is a local maximizer. This is because $P\left(\Delta, \alpha, \frac{\alpha}{\beta}\right)$ is convex in $\Delta$ for small $\Delta$ if (13) holds which gives rise to the possibility of multiple stationary points. However, there are decreasing returns to increasing $\Delta$ over and above $\underline{\Delta}$ for $\beta \in\left(0, \beta^{\prime \prime}\right]$, by essentially the same argument as above. Therefore, the smallest local maximizier is the unique global maximizer 
in this range.

To summarize this discussion, exposing the agent to extreme options is attractive from the cost side if the agent's effort choice under full freedom of action is already quite high. In this case the agent is almost never forced to make up his mind if his information is coarse. From the benefit side, extreme options are attractive as an incentive device if the agent responds well to incentives, which is the case if his effort choice under full discretion is bounded away from the extremes. Under the conditions we provide the net benefit is largest for some intermediate level of effort.

One may wonder how $\alpha$ influences the principal's optimal choice of $\Delta$. Unfortunately results in this vein are ambiguous. The reason is that $\alpha$ determines at the same time the weight the principal attaches to the agent's utility in joint utility and the level of effort the agent is willing to exert for any contract. This gives rise to sometimes countervailing effects and therefore ambiguous comparative statics. In the remainder of this section we shall therefore drop some of our assumptions to resolve this ambiguity. As we shall not need the multiplicative form of the cost-of-effort function for this and the remaining analysis we shall set $\beta \equiv 1$ and identify the functions $g(e)$ and $h(e)$ in what follows.

Since $e(\Delta, \alpha)$ is monotone increasing in $\alpha$ our previous results are exactly reversed in any situation where the weight the principal attaches to the agent's utility in joint utility does not depend on $\alpha$. In particular, this is true if the principal can neglect any costs she inflicts on the agent.

Proposition 7 Suppose that the agent is already in place, his compensation package determined by an outside process and let his IR constraint be nonbinding. Suppose further that $g(e)$ is a general INADA cost function satisfying $\lim _{e \rightarrow 1} \frac{g_{e e e}(e)}{g_{e e}(e)^{2}}>2$ and $\frac{g_{e}(e)}{g_{e e}(e)}$ concave in $e$. Then, the qualitative features of proposition 6 remain intact with $\beta$ replaced by $\alpha^{-1}$.

One easily verifies that the first order condition for an optimal solution for the case of a nonbinding IR constraint is

$$
\left.\left(\left(e(\Delta, \alpha)+\frac{g_{e}(e(\Delta, \alpha))}{g_{e e}(e(\Delta, \alpha))}\right)\left(E \pi_{\Delta}(\Delta, \phi)-E \pi_{\Delta}(\Delta, \eta)\right)-\Delta\right)\right|_{\Delta_{=\Delta^{*}}}=0
$$

which makes proposition 6 obvious.

Finally, for the case of a binding IR constraint, one may simply assume that the net benefit of small restrictions is monotonic in $\alpha$. This amounts to dropping the INADA condition that $\lim _{e \rightarrow 1} g_{e}(e)=\infty$. Clearly this implies that an optimal contract satisfying the first order condition ((15) with $\beta=1)$ must be monotonic in $\alpha$. However, for $\alpha$ high enough the contract eventually 
implements acquisition of precise information with probability 1 . In this range of parameter values the principal can decrease the size of the prohibited set and still implement the same marginal value of information if $\alpha$ is increased. These results are summarized in the following proposition.

Proposition 8 Suppose the agent's IR constraint is binding. Suppose further that $\lim _{e \rightarrow 1} g_{e}(e)<$ $\infty$ and let $\frac{g_{e}(e)}{g_{e e}(e)}$ be nondecreasing in $e^{13}$. Then,

there exist two strictly positive and bounded values of the parameter $\alpha, \alpha^{\prime}$ and $\alpha^{\prime \prime}$, (defined explicity in the appendix) satisfying $\alpha^{\prime \prime}>\alpha^{\prime}>0$, such that

i) $\triangleq$ is equal to zero for $\alpha \leq \alpha^{\prime}$, increasing in $\alpha$ for $\alpha^{\prime}<\alpha \leq \alpha^{\prime \prime}$, and decreasing in $\alpha$ for $\alpha>\alpha^{\prime \prime}$.

ii) $e(\underline{\Delta}, \alpha)$ is monotone nondecreasing in $\alpha$ for all $\alpha . e(\underline{\Delta}, \alpha)$ is equal to 1 for all $\alpha \geq \alpha^{\prime \prime}$.

iii) If $\alpha \geq \alpha^{\prime \prime}$ then $\underline{\Delta}=\Delta^{*}$.

Propositions 7 and 8 lend further support for our earlier conclusions because the qualitative features of the optimal contract are robust to variations in the model setup. Crucial to this robustness of our comparative statics results is the success enhancing technology. For these technologies the cost of clear advice is zero for $e$ close to one. Moreover, any regularity condition -which is consistent with at least one of the boundary conditions of INADA functions - imposed on the value of the agent's responsiveness produces the result that the benefit of extreme options outweigh the cost if the agent's choice of effort is close to 1 .

\section{Monetary Sanctions and Motivation}

We now turn to the opposite extreme when the agent is risk neutral with respect to income shocks. In this case results depend crucially on whether performance and messages are contractible or whether only messages are contractible. In the former case, which has been studied among other problems by Osborne (1985), it is possible to implement the first best. The solution involves a transfer that reflects the principal's payoff, i.e., a transfer scheme $t(x(\hat{\eta}), \eta)=\bar{t}-\pi(x(\hat{\eta}), \eta)$. Confronted with this transfer the agent has the correct incentive to acquire information as well as to reveal information truthfully and first best is achieved. The result obtains because the agent has unlimited wealth (and is risk neutral with respect to money payments). We study the case where performance is noncontractible. The standard justification for this assumption is that performance

\footnotetext{
${ }^{13}$ The property $\frac{g_{e}(e)}{g_{e e}(e)}$ is possessed by convex power functions, $e^{n}$, exponentials, exp $(e)$, and products of these two classes. Sums of (two) powerfunctions $e^{n}+e^{l}$ display the property provided that $|n-l|$ is small. As an example consider a quadratic cost function and let $\frac{\sigma^{2}}{2}=1$. One easily verifies that (13) holds if $1>\alpha>\frac{\sqrt{5}-1}{2}$.
} 
is observable but not verifiable. ${ }^{14}$

By the revelation principle we can restrict attention to direct mechanisms that give the agent the incentive to tell the truth. The principal's problem is a straightforward extension of problem (5) s.t. (6) - (9) with the additional complication that transfers may depend on the agent's recommendation. We let $\{x(\eta), t(\eta)\}$ denote the contract tuple offered to the agent in case he announces that the experiment was a success and that the realization of $\tilde{\eta}$ was $\eta$. $\{x(\phi), t(\phi)\}$ denotes the contract offered to the agent in case he announces that the experiment failed. Formally, the principal's problem is:

$$
\begin{gathered}
\max _{\substack{x(\eta), t(\eta), x(\phi), t(\phi), e}}(1-e) E[V(x(\phi), \tilde{\eta})-t(\phi)]+e E[V(x(\eta), \tilde{\eta})-t(\eta)] \\
\text { s.t. } \\
\forall \eta: U(x(\eta), \eta, \alpha)+t(\eta) \geq U(x(\phi), \eta, \alpha)+t(\phi) \\
\forall \eta: \quad U(x(\eta), \eta, \alpha)+t(\eta) \geq U(x(\hat{\eta}), \eta, \alpha)+t(\hat{\eta}) \quad \forall \hat{\eta} \\
E[U(x(\phi), \tilde{\eta}, \alpha)+t(\phi)] \geq E[U(x(\hat{\eta}), \tilde{\eta}, \alpha)+t(\hat{\eta})] \quad \forall \hat{\eta} \\
E[U(x(\eta), \tilde{\eta}, \alpha)+t(\eta)]-E[U(x(\phi), \tilde{\eta}, \alpha)+t(\phi)]=g_{e}(e) \\
(1-e) E[U(x(\phi), \tilde{\eta}, \alpha)+t(\phi)]+e E[U(x(\eta), \tilde{\eta}, \alpha)+t(\eta)] \geq g(e)
\end{gathered}
$$

The agent is given incentives to announce truthfully the failure of the experiment (18), and to announce truthfully the true realization of $\eta$ if he knows it rather than any other realization of $\tilde{\eta}$ or that the experiment failed (17). The agent's effort choice is determined by the marginal value of information the contract provides (19) and the agent is willing to participate (20). It is immediate that no contract of this restricted class can implement the first best:

Lemma 2: There exists no contract which is ex post and ex ante efficient.

Ex post efficiency requires that the agent receives the same transfer for all values of $\eta$ he announces, given that he announces that the experiment was a success. To see this, consider local incentive compatibility of the agent's announcement conditional on a successful experiment, i.e., $\alpha(x(\hat{\eta})-\eta) \frac{\partial x(\hat{\eta})}{\partial \hat{\eta}}=\frac{\partial t(\hat{\eta})}{\partial \hat{\eta}}$. Ex post efficiency requires that the left hand side of this equation is zero. By consequence the transfer must be flat where it is differentiable. From global incentive compatibility, one observes that $t(\hat{\eta})$ must be continuous, because otherwise some types would

\footnotetext{
${ }^{14}$ For a different justification of message contingent contracts in a similar context, see Prendergast (1993).
} 
have an incentive to misrepresent their types. Since $x(\phi)=x(\mu)=\mu$ in an ex post efficient contract, this leaves only one possibility to give the agent more of an incentive to exert effort: to set $t(\phi)$ sufficiently smaller than $t(\mu)$. However, if the same decision $x$ is taken for reports $\phi$ and $\mu$, the agent can always claim to be the type that would receive the higher transfer. But then, the transfer scheme can't be used at all to give the agent more of an incentive to exert effort. Consequently, first best cannot be achieved by any contract and conversely, giving extra incentives for information acquisition implies a departure from ex post efficient use of information.

By quasilinearity of utilities the agent's individual rationality constraint is binding at the optimum and the principal maximizes joint surplus subject to incentive compatibility of the agent's choices. The solution procedure essentially parallels the one presented in section 3. To avoid duplication we directly present the solution. ${ }^{15}$

Proposition 9 Suppose that $\alpha \in(0, \infty)$. Then the principal implements an ex post inefficient contract. Suppose that $\frac{\partial f(\eta)}{\partial \eta} \geq 0$ for $\eta \leq \mu$ and that $\frac{\partial f(\eta)}{\partial \eta} \leq 0$ for $\eta>\mu$ and that $g_{\text {eee }}(e) \geq 0 \forall e$. Then, the optimal contract has the following properties:

a) The action $x(\phi)$ is ex post efficient. The function $x(\eta)$ is strictly increasing with a discontinuity at the mean. There is pooling of types $\mu$ and $\phi$. Formally,

$$
\begin{aligned}
& x(\phi)=\mu \\
& x(\eta)=\eta-\gamma(\alpha) \frac{F(\eta)}{f(\eta)} \text { for } \eta<\mu \\
& x(\mu)=\mu \\
& x(\eta)=\eta+\gamma(\alpha) \frac{1-F(\eta)}{f(\eta)} \text { for } \eta>\mu .
\end{aligned}
$$

b) The transfer scheme is decreasing in $\eta$ for $\eta \leq \mu$, increasing in $\eta$ for $\eta>\mu$, and displays a

\footnotetext{
${ }^{15} 1_{\Phi}$ is equal to 1 if the statement in $\Phi$ is true, and zero if not. $T$ is the optimal indirect utility level given to type $\underline{\eta}, u^{*}(\underline{\eta})$. One derives it from the binding IR constraint. $T=g(e)-A K-(1-e) \frac{A \sigma^{2}}{2}+$ $A \gamma(e)\left\{(1-e) \int_{\underline{\eta}}^{\mu} \frac{F(\eta)}{f(\eta)} d \eta+e \int_{\underline{\eta}}^{\bar{\eta}} \frac{\left(1_{\eta>\mu}-F(\eta)\right)^{2}}{f(\eta)} d \eta\right\}$.
} 
discontinuity at the mean. Formally,

$$
\begin{aligned}
& t(\phi)=T-\alpha \gamma(\alpha) \int_{\underline{\eta}}^{\mu} \frac{F(z)}{f(z)} d z \\
& t(\eta)=T+\frac{\alpha}{2}\left(\gamma(\alpha) \frac{F(\eta)}{f(\eta)}\right)^{2}-\alpha \gamma(\alpha) \int_{\underline{\eta}}^{\eta} \frac{F(z)}{f(z)} d z \quad \text { for } \eta<\mu \\
& t(\mu)=T-\alpha \gamma(\alpha) \int_{\underline{\eta}}^{\mu} \frac{F(z)}{f(z)} d z \\
& t(\eta)=T+\frac{\alpha}{2}\left(\gamma(\alpha) \frac{1-F(\eta)}{f(\eta)}\right)^{2}+\alpha \gamma(\alpha) \int_{\underline{\eta}}^{\eta} \frac{1_{z>\mu}-F(z)}{f(z)} d z \text { for } \eta>\mu
\end{aligned}
$$

where

$$
\gamma(\alpha)=\frac{\frac{g_{e}\left(e^{*}\right)}{\alpha}-\frac{\sigma^{2}}{2}}{\int_{\underline{\eta}}^{\bar{\eta}} \frac{\left(1_{\eta>\mu}-F(\eta)\right)^{2}}{f(\eta)} d \eta}
$$

c) $e^{*}$ is uniquely defined by the equation

$$
\left.\left(\frac{\frac{g_{e}(e)}{\alpha}-\frac{\sigma^{2}}{2}}{\int_{\underline{\underline{\eta}}}^{\bar{\eta}} \frac{\left(1_{\eta>\mu}-F(\eta)\right)^{2}}{f(\eta)} d \eta}\right)\right|_{e=e^{*}}=\left.\left(\frac{\frac{\alpha+1}{2} \sigma^{2}-g_{e}(e)}{\frac{1+\alpha}{2}\left(\frac{g_{e}(e)}{\alpha}-\frac{\sigma^{2}}{2}\right)+e(\alpha) \frac{1+\alpha}{\alpha} g_{e e}(e)}\right)\right|_{e=e^{*}}
$$

For $\alpha$ smaller than some critical value (defined in the appendix) $e^{*}$ is optimal in the local sense. For $\alpha$ large $e^{*}$ is unique and optimal in the global sense.

Figure 1 provides a graphical illustration of the optimal contract. The principal now has a richer set of incentive instruments at hand to make experiment failure relatively unattractive to the agent. By (19)the marginal value of information to the agent is increased c.p. if i) the course of action conditional on experiment failure is distorted away from $\mu$ so as to increase the difference between the agent's expected utility level conditional on experiment failure and his expected loss conditional on a successful experiment, and/or if ii) the transfer conditional on experiment failure is decreased relative to the expected payment conditional on a successful experiment. Moreover, the choice of action and the transfer, both conditional on experiment failure, are substitute incentive instruments in the sense that any expected utility level conditional on experiment failure can be implemented either by a high financial sanction and an efficient choice of act or by a lower sanction and a distorted choice of act. Therefore, it is not necessary to implement an inefficient choice of act if the experiment fails in order to give the agent an added incentive to acquire information.

With this enriched set of incentive instruments it is always preferred to use the transfer scheme rather than the decision scheme to make experiment failure unattractive relative to success, i.e., any efficient contract involves $x(\phi)=\mu$. To see this result observe that any contract must involve 


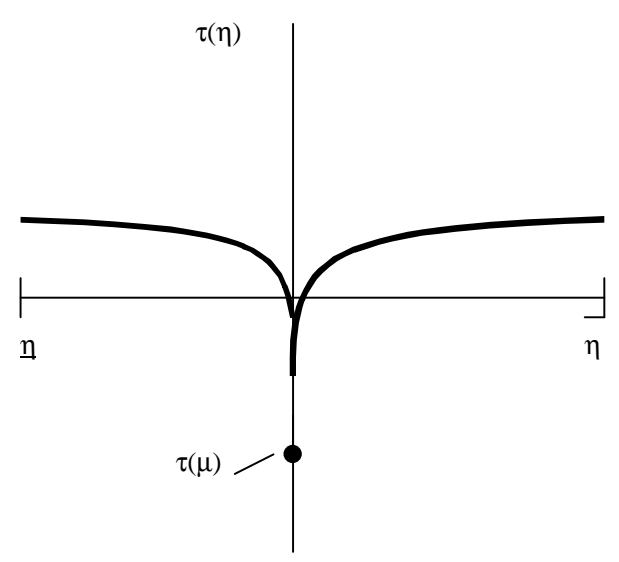

b)

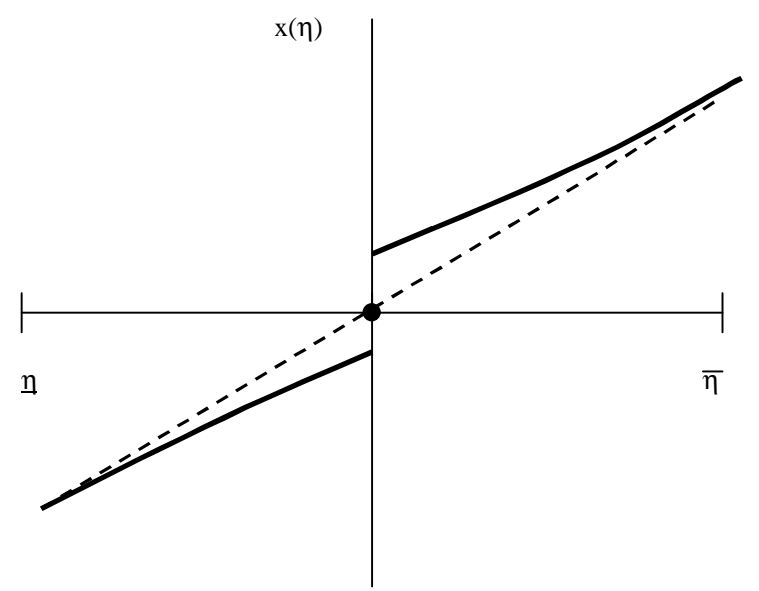

Figure 1: Diagram a) is the transfer scheme. The agent's financial reward is the higher the farther his type from the mean. Diagram b) depicts the optimal choice function $x(\cdot)$. At the mean, the ex post efficient act is implemented.

pooling of types $\phi$ and $\mu$. These types share the same conditional expected value of $\tilde{\eta}$. Therefore their preferences over contracts $\{x(\phi), t(\phi)\}$ and $\{x(\mu), t(\mu)\}$ coincide for any contract the principal offers. This pooling property allows us to derive the endogenous ex ante distribution of types: any type $\eta \neq \mu$ has ex ante likelihood ef $(\eta)$, while type $\mu$ occurs with ex ante probability $1-e+e f(\mu)$. Therefore the ex ante distribution of types effectively has a mass point at the mean. It follows that it is infinitely more costly to implement a distorted choice of act conditional on experiment failure than to distort the decision scheme $x(\eta)$ for any type different from $\mu$. By consequence it is always optimal to implement the efficient choice of act conditional on experiment failure and use the transfer scheme instead of the decision schedule to make experiment failure relatively unattractive to the agent.

Except in the trivial cases it is now always optimal to give the agent more of an incentive to acquire information. This is precisely because the optimal contract involves no efficiency loss in case the experiment fails. Therefore the ex post cost of introducing distortions is smaller by an order of magnitude than the ex ante gain arising through the beneficial effect on the agent's incentive to acquire more information.

Consider now the distorted part of the decision schedule $x(\eta)$. Let $\mu^{+}=\inf \eta \mid \eta>\mu$ and $\mu^{-}=\sup \eta \mid \eta<\mu$. If the agent is punished financially contingent on experiment failure, the truth 
telling constraint of type $\phi$ implies that $x\left(\mu^{+}\right)$and $x\left(\mu^{-}\right)$must be distorted away from ex post efficiency. This way, type $\phi$ is confronted with a choice between the ex post efficient act and a low transfer if he tells the truth and an inefficient choice of act $x\left(\mu^{+}\right)\left(x\left(\mu^{-}\right)\right)$together with a relatively higher transfer in case he claims to be of type $\mu^{+}\left(\mu^{-}\right)$. By local incentive compatibility of the contract the distortion spreads out over the whole support of the distribution with the bounds being an exception. The size of the distortion at a given type $\eta$ reflects a trade-off between increasing the joint ex post loss and increasing the agent's marginal information rent.

Under the standard conditions on hazard rates $\frac{\partial}{\partial \eta} \frac{F(\eta)}{f(\eta)} \leq 1$ for $\eta<\mu$ and $\frac{\partial}{\partial \eta} \frac{1-F(\eta)}{f(\eta)} \geq-1$ for $\eta>\mu$, which are equivalent to the stated condition on the density, the contract is strictly monotonic so that there is no bunching. Moreover, under relatively mild additional conditions on $g(\cdot)$ and $\alpha$ the principal faces a concave problem with a unique solution for large enough $\alpha$. Unfortunately, however, the solution is too involved to allow for a comparative statics analysis.

It is interesting to contrast the optimal contract with properties of contracts found in similar problems. Our contract does not have the property of no distortion at the top. Instead the contract has "no distortion at the bottom" and "distortions at the top a.e.". With the usual ordering of types according to ex post efficiency the bottom corresponds to type $\phi$. The top in contrast corresponds to all remaining types. The top is undistorted for type $\mu$ and at the bounds $\underline{\eta}$ and $\bar{\eta}$. In the first case this is because there is pooling of types $\mu$ and $\phi$. In the later case this is because the concern for increasing the agent's marginal information rent is absent at the bounds of the support. Finally, there is no bunching in the contract. ${ }^{16}$

The second best contract does no longer allow for the interpretation that the agent is forced to depart from the prior optimal choice. In case he announces to not have acquired new information, the efficient course of action is followed. However, quite similar to the case without transfers, the principal goes too far in following the agent's advice for some reports: it is still optimal to implement $x(\eta)>\eta$ for $\eta>\mu$ and $x(\eta)<\eta$ for $\eta<\mu$, except at the bounds of the support. Some actions are never taken in equilibrium, as the contract is discontinuous at the mean. However, the

\footnotetext{
${ }^{16}$ This is interesting from a technical perspective, because Lewis and Sappington (1993) have a result going in the opposite direction. The reason for this discrepancy is that our agent is benevolent in contrast to theirs. As here, in their procurement problem, the possibility of ignorance induces a strong desire for implementing the efficient course of action in case the agent confesses ignorance and the principal is lead to increase the quantity bought towards the efficient amount. However, this desire runs counter the desire to limit the agent's informational rents which induces the principal to buy less than would be efficient. In our problem, there is no conflict between these goals as the optimal choice is distorted downwards for $\eta<\mu$ and upwards for $\eta>\mu$. Consequently, the efficient choice at the mean is not in conflict with monotonicity in the contract and there is no bunching under the usual conditions.
} 
set of actions the agent is "prohibited from taking" is no longer convex but rather consists of two convex sets separated by the isolated point, $\mu$.

We conjecture that similar results could be obtained under different assumptions. For instance if payoffs were contractible, but the agent had limited liability (e.g., Lewis and Sappington (1997), Crémer et. al (1998)). ${ }^{17}$

\section{Extensions}

One can generalize the insights of the model with infinite risk aversion with respect to money income in a number of directions.

One may allow for alternative location experiments and let the agent have a normally distributed prior about the true state of the world, $\eta$, and have him acquire a normally distributed signal, whose determininistic precision is increasing in effort. Some of our results are robust to this extension some are not. In particular, when the agent is unable to choose acts in a symmetric, convex set around the prior optimal act he finds it more attractive to acquire more precise information. To see why, observe that the agent would like to choose $x$ ex post equal to (or as close as possible to) his conditional expected value of $\eta$. As is well known for normal location experiments (see, e.g., deGroot (1970)), the conditional expectation is a weighted average of the prior mean and the signal, with the weight on the signal being the higher the higher its precision. From the ex ante perspective the conditional expectation function is itself a normally distributed random variable. Its variance is the higher the higher the agent's effort level. On the one hand the signal is more precise, which tends, c.p., to decrease the variance in the ex ante distribution. On the other hand the agent puts more weight on the signal relative to the prior in forming the conditional expectation which tends to increase the variance in the ex ante distribution. For normal location experiments the second effect dominates the first so that it is the less likely that the agent wants to choose an act close to the prior optimal act the more effort the agent exerts. By consequence, a higher effort helps the agent to avoid the punishment so that clear advice can again be used as an incentive device. Moreover, the principal may benefit from using the incentive instrument. It proves very

\footnotetext{
${ }^{17}$ As a referee pointed out, first best would be implementable by a Crémer McLean type mechanism if the principal also acquired information with a certain probability. Then, the principal could give the agent a very low financial transfer if he announced some type $\eta=\eta_{1}$ while in fact his experiment was a failure but the principal's succeeded and revealed that $\eta=\eta_{2} \neq \eta_{1}$. By consequence, $\tau(\phi)$ can be decreased relative to $\tau(\eta)$ for all $\eta$, while $x(\eta)=\eta$ is implemented for all $\eta$ without giving the agent an incentive to misrepresent his type.
} 
difficult to characterize the optimal choice set for normal location experiments. The reason is that the agent's effort no longer shifts mass away from a mass point but rather from a whole set around the prior optimal act. The possibilities to provide incentives are much richer than for success enhancing technologies. Interestingly, in an example we provide, the principal finds it optimal to force the agent to give clear advice when the agent's taste parameter, $\alpha$ is low. This shows how important the success enhancing technology is for our comparative statics results in section 3 .

Consider the role of identical preferences ex post. Two points should be made here. First, the formulation is more general than the case of identical partisan objectives. Our application to portfolio management (Szalay 2003) makes evident that congruence of ex post objectives arises naturally in case the agent does not care for performance per se but receives a payment which is linear in performance. ${ }^{18}$ Second, one may allow for diverging partisan objectives. In Szalay (2000) we consider the case where the agent's bias is not known to the principal, but where the principal knows that the agent's preferred act is correlated with hers. If the correlation is large enough, the principal gains from prohibiting the agent from choosing the prior optimal act. If the correlation is low the agent's information is of no use to the principal since he would not use it in the principal's interest. Therefore, no extra incentives are provided by banning the prior optimal act and acts close to it. However, in this case the principal benefits from constraining the agent's right to choose extreme acts. The optimal extreme bounds on the agent's choice set display the Aghion-Tirole trade-off of initiative versus loss of control.

Finally, in Szalay (2001) we have considered a model where the agent faces the discrete choice of whether to innovate or not and have shown that our main results are robust with respect to allowing for discreteness. In particular, we consider the choice between a risky and a safe option, e.g., a manager choosing between an uncertain innovation and the safe status quo. The manager screens by exerting effort among innovation paths. Imposing an innovation bias may increase ex ante expected payoffs although the manager sometimes has to gamble because he puts in more effort to screen the innovation paths.

\section{Conclusion}

We have studied freedom of choice as an incentive device. The main point of the present paper is that the inability to choose can be used to provide incentives for information acquisition. Forced

\footnotetext{
${ }^{18}$ See also Stoughton (1993) on this.
} 
to choose among extreme options, or equivalently, forced to give clear advice, an agent finds it relatively more attractive to be well informed. A number of applications of this idea are discussed: judicial decision-making, delegated portfolio management (see our companion paper Szalay (2003)), and activism in organizations. More generally, our theory applies to situations where there is perfect commitment to the rules of decision-making and no conflict of interest ex post between the contracting parties. Moreover, as we have shown in Szalay (2000) the theory can be extended to cover situations with conflicts of interest ex post. We have not discussed multiagent situations. One interesting possibility would be to study rules of arbitration, as analyzed, e.g., by Gibbons (1988) from the perspective of providing incentives to the disputing parties to make reasonable offers, from the perspective of providing incentives for effort to the arbitrator. Our analysis suggests that eliminating the possibility for compromise may be good for the arbitrator's incentive to find out which of two opposing positions is closer to the truth. We believe this is an interesting avenue for research and leave it to future work.

\section{Appendix A}

Proof of Proposition 1. By quasilinearity of payoffs and unlimited wealth the principal maximizes joint payoff subject to incentive compatibility of the agent's choices. The principal's payoff function can be written as

$$
\begin{aligned}
& E W(x(\phi), \tilde{\eta}, \alpha)+e(E W(x(\eta), \tilde{\eta}, \alpha)-E W(x(\phi), \tilde{\eta}, \alpha))-g(e) \\
= & E W(x(\phi), \tilde{\eta}, \alpha)+e \frac{1+\alpha}{\alpha}(E U(x(\eta), \tilde{\eta}, \alpha)-E U(x(\phi), \tilde{\eta}, \alpha))-g(e) \\
= & E W(x(\phi), \tilde{\eta}, \alpha)+e \frac{1+\alpha}{\alpha} g_{e}(e)-g(e)
\end{aligned}
$$

where the first equality uses (2) and the second equality uses (8). We observe that, for a given $x(\phi)$, the principal's payoff is increasing in $e$ because $e \frac{1+\alpha}{\alpha} g_{e}(e)-g(e)$ is strictly increasing in $e$. From (8) one observes that in turn $e$ is strictly increasing in $E U(x(\eta), \tilde{\eta}, \alpha)$ for a given $E U(x(\phi), \tilde{\eta}, \alpha)$. Therefore, taking $|x(\phi)-\mu|=\Delta$ as given an optimal contract solves

$$
\begin{gathered}
\max _{\Gamma} E U(x(\eta), \tilde{\eta}, \alpha) \\
\text { s.t. }|x(\phi)-\mu|=\Delta
\end{gathered}
$$


The solution to this problem is

$$
\begin{aligned}
& \Gamma=[\underline{\eta}, \bar{\eta}] \backslash(\mu-\Delta, \mu+\Delta) \quad \text { for } \Delta \in[0, \min \{\mu-\underline{\eta}, \bar{\eta}-\mu\}] \\
& =\quad[\mu+\Delta, \bar{\eta}] \quad \text { for } \Delta>\min \{\mu-\underline{\eta}, \bar{\eta}-\mu\} \quad \text { if } \bar{\eta}-\mu>\mu-\underline{\eta} \\
& =[\underline{\eta}, \mu-\Delta] \quad \text { for } \Delta>\min \{\mu-\underline{\eta}, \bar{\eta}-\mu\} \quad \text { if } \bar{\eta}-\mu<\mu-\underline{\eta}
\end{aligned}
$$

It is unique. Suppose not and there are two closed sets that solve this problem. Since both achieve the same value of the objective they can differ only on measure zero sets. By full support of $f(\eta)$ measure zero sets are isolated points. But then at least one of the sets is not closed, a contradiction. Finally, $\Delta^{*}<\min \{\mu-\underline{\eta}, \bar{\eta}-\mu\}$. We shall prove this for the case $\mu-\underline{\eta}<\bar{\eta}-\mu$. The proof of the reverse case is analogous and omitted. Consider the incremental loss. By straightforward algebra

$$
\begin{aligned}
E \pi(x(\phi), \tilde{\eta})-E \pi(x(\eta), \tilde{\eta})-= & \begin{array}{cc}
\frac{1}{2}\left(\sigma^{2}+\Delta^{2}\right)-\frac{1}{2} \int_{\mu}^{\mu+\Delta}(\mu+\Delta-\eta)^{2} d F(\eta) \\
\end{array} \\
=\frac{1}{2} \int_{\mu-\Delta}^{\mu}(\mu-\Delta-\eta)^{2} d F(\eta) & \text { if } \Delta<\mu-\underline{\eta}
\end{aligned}
$$

By straightforward calculus one finds that $\left(\sigma^{2}+\Delta^{2}\right)-\int_{\underline{\eta}}^{\mu+\Delta}(\mu+\Delta-\eta)^{2} d F(\eta)$ is decreasing convex in $\Delta$ (with slope zero at $\Delta=\bar{\eta}-\mu$ ). Moreover, one can show that

$$
\begin{aligned}
& \lim _{\Delta \nearrow \mu-\underline{\eta}} \Delta^{2}-\int_{\mu}^{\mu+\Delta}(\mu+\Delta-\eta)^{2} d F(\eta)-\int_{\mu-\Delta}^{\mu}(\mu-\Delta-\eta)^{2} d F(\eta) \\
> & \lim _{\Delta \searrow \mu-\underline{\eta}} \Delta^{2}-\int_{\underline{\eta}}^{\mu+\Delta}(\mu+\Delta-\eta)^{2} d F(\eta)
\end{aligned}
$$

Hence, the marginal value of information is always higher if the agent has at least one option on either side rather than when not.

Proof of Proposition 2. From proposition 1 the difference between expected losses conditional on experiment failure and conditional on success is

$$
E \pi(\Delta, \phi)-E \pi(\Delta, \eta)=\frac{\left(\sigma^{2}+\Delta^{2}\right)}{2}-\frac{\int_{\mu}^{\mu+\Delta}(\mu+\Delta-\eta)^{2} d F(\eta)+\int_{\mu-\Delta}^{\mu}(\mu-\Delta-\eta)^{2} d F(\eta)}{2}
$$

and the agent's effort choice satisfies:

$$
e(\Delta, \alpha)=g_{e}^{-1}\left[\frac{\alpha}{2}\left(\sigma^{2}+\Delta^{2}\right)-\frac{\alpha}{2} \int_{\mu}^{\mu+\Delta}(\mu+\Delta-\eta)^{2} d F(\eta)-\frac{\alpha}{2} \int_{\mu-\Delta}^{\mu}(\mu-\Delta-\eta)^{2} d F(\eta)\right]
$$

By Leibniz's rule and the inverse function theorem

$$
e_{\Delta}(\Delta, \alpha)=\frac{\alpha}{g_{e e}(e)}\left\{\Delta-\int_{\mu}^{\mu+\Delta}(\mu+\Delta-\eta) d F(\eta)+\int_{\mu-\Delta}^{\mu}(\mu-\Delta-\eta) d F(\eta)\right\}
$$

Hence, $e_{\Delta}(\Delta, \alpha)>0$ iff $\Delta-\int_{\mu}^{\mu+\Delta}(\mu+\Delta-\eta) d F(\eta)+\int_{\mu-\Delta}^{\mu}(\mu-\Delta-\eta) d F(\eta)>0$. Let now $\mu+Z^{+}(\Delta):=E[\eta \mid \eta \in[\mu, \mu+\Delta]]$ and $\mu-Z^{-}(\Delta):=E[\eta \mid \eta \in[\mu-\Delta, \mu]]$. Using these definitions 
one can rewrite

$$
\begin{aligned}
& \Delta-\int_{\mu}^{\mu+\Delta}(\mu+\Delta-\eta) d F(\eta)+\int_{\mu-\Delta}^{\mu}(\mu-\Delta-\eta) d F(\eta) \\
& =\Delta-(F(\mu+\Delta)-F(\mu-\Delta)) \Delta+(F(\mu+\Delta)-F(\mu)) Z^{+}(\Delta)+(F(\mu)-F(\mu-\Delta)) Z^{-}(\Delta) \\
& \geq(F(\mu+\Delta)-F(\mu)) Z^{+}(\Delta)+(F(\mu)-F(\mu-\Delta)) Z^{-}(\Delta) \\
& \geq 0
\end{aligned}
$$

where the last inequality is strict if $\Delta>0$, because $Z^{+}(\Delta)$ and $Z^{-}(\Delta)$ are strictly positive by construction.

Proof of Proposition 3. Let

$$
P(\Delta, \alpha)=E W(x(\phi), \tilde{\eta}, \alpha)+e \frac{1+\alpha}{\alpha} g_{e}(e)-g(e)
$$

denote the principal's payoff function. $P(\Delta, \alpha)$ is a continuous function of $\Delta . \Delta$ is chosen from the interval $[0, \mu-\underline{\eta}$. By Weierstrass' theorem a continuous function on a compact domain must attain a maximum. Differentiating $P(\Delta, \alpha)$ with respect to $\Delta$, using $(23)$ and the envelope theorem, one obtains

$$
P_{\Delta}(\Delta, \alpha)=\left(e(\cdot)(1+\alpha)+\frac{g_{e}(e(\cdot))}{g_{e e}(e(\cdot))}\right)\left(E \pi_{\Delta}(\Delta, \phi)-E \pi_{\Delta}(\Delta, \eta)\right)-\Delta(1+\alpha)
$$

More specifically, using (21), one finds that

$$
P_{\Delta}(\Delta, \alpha)=\left(e(\cdot)(1+\alpha)+\frac{g_{e}(e(\cdot))}{g_{e e}(e(\cdot))}\right)\left\{\begin{array}{c}
\Delta-\int_{\mu}^{\mu+\Delta}(\mu+\Delta-\eta) d F(\eta) \\
+\int_{\mu-\Delta}^{\mu}(\mu-\Delta-\eta) d F(\eta)
\end{array}\right\}-\Delta(1+\alpha)
$$

and observes that $\left.\frac{\partial P(\Delta, \alpha)}{\partial \Delta}\right|_{\Delta=0}=0$. Hence $\Delta=0$ is a stationary point. Differentiating a second time one obtains

$$
\begin{aligned}
P_{\Delta \Delta}(\Delta, \alpha)= & -1(1+\alpha)+\left(e(\cdot)(1+\alpha)+\frac{g_{e}(e(\cdot))}{g_{e e}(e(\cdot))}\right)\left(E \pi_{\Delta \Delta}(\Delta, \phi)-E \pi_{\Delta \Delta}(\Delta, \eta)\right) \\
& +\frac{\partial}{\partial e}\left(e(\cdot)(1+\alpha)+\frac{g_{e}(e(\cdot))}{g_{e e}(e(\cdot))}\right) \frac{\alpha}{g_{e e}(e(\cdot))}\left(E \pi_{\Delta}(\Delta, \phi)-E \pi_{\Delta}(\Delta, \eta)\right)^{2}
\end{aligned}
$$

Using (21) once more

$$
\begin{gathered}
P_{\Delta \Delta}(\Delta, \alpha)=-1(1+\alpha)+\left(e(\cdot)(1+\alpha)+\frac{g_{e}(e(\cdot))}{g_{e e}(e(\cdot))}\right)\left\{1-\int_{\mu}^{\mu+\Delta} d F(\eta)-\int_{\mu-\Delta}^{\mu} d F(\eta)\right\} \\
+\frac{\partial}{\partial e}\left(e(\cdot)(1+\alpha)+\frac{g_{e}(e(\cdot))}{g_{e e}(e(\cdot))}\right) \frac{\alpha\left\{\Delta-\int_{\mu}^{\mu+\Delta}(\mu+\Delta-\eta) d F(\eta)+\int_{\mu-\Delta}^{\mu}(\mu-\Delta-\eta) d F(\eta)\right\}^{2}}{g_{e e}(e(\cdot))}
\end{gathered}
$$


Evaluating this expression at $\Delta=0$ gives

$$
\left.P_{\Delta \Delta}(\Delta, \alpha)\right|_{\Delta=0}=-1(1+\alpha)+\left.\left(e(\Delta, \alpha)(1+\alpha)+\frac{g_{e}(e(\Delta, \alpha))}{g_{e e}(e(\Delta, \alpha))}\right)\right|_{\Delta=0}
$$

Hence if $\left.P_{\Delta \Delta}(\Delta, \alpha)\right|_{\Delta=0}>0, \Delta=0$ is a local minimum.

Proof of Proposition 4. Let

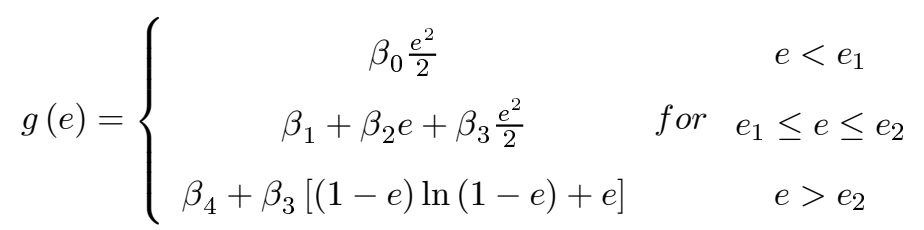

where $e_{1}=\frac{\beta_{2}}{\beta_{0}-\beta_{3}}$ and $e_{2}$ is the the positive solution to $\beta_{2}+\beta_{3} e=-\beta_{3} \ln (1-e)$. It is immediate that i) $e_{2} \in(0,1)$ for any strictly positive and bounded values of $\beta_{2}$ and $\beta_{3}$ and ii) that for any values of $\beta_{2}$ and $\beta_{3}$, there exists a $\beta_{0}$ large enough such that $e_{1} \in\left(0, e_{2}\right)$. Moreover, since $e_{1}$ and $e_{2}$ are independent of $\beta_{1}$ and $\beta_{4}$, we can choose $\beta_{1}$ such that $\beta_{0} \frac{e_{1}^{2}}{2}=\beta_{1}+\beta_{2} e_{1}+\beta_{3} \frac{e_{1}^{2}}{2}$ and $\beta_{4}$ such that $\beta_{1}+\beta_{2} e_{2}+\beta_{3} \frac{e_{2}^{2}}{2}=\beta_{4}+\beta_{3}\left[\left(1-e_{2}\right) \ln \left(1-e_{2}\right)+e_{2}\right]$. This construction has $g(e)$ and $g_{e}(e)$ continuous and $g_{e}(e)$ differentiable. For any finite $\beta_{0}$ and any positive $\beta_{3}, g(e)$ is a convex INADA function.

To ease notation in this proof define $\hat{e}:=\left.(e(\Delta, \alpha))\right|_{\Delta=0}$. Suppose, by way of a working hypothesis, that $\hat{e} \in\left[e_{1}, e_{2}\right]$. Then, from $\left.\left(g_{e}(e)\right)\right|_{e=\hat{e}}=\frac{\alpha \sigma^{2}}{2}$, the parameters $\beta_{2}$ and $\beta_{3}$ must satisfy $\beta_{2}=$ $\frac{\alpha \sigma^{2}}{2}-\hat{e} \beta_{3}$. To satisfy the working hypothesis, we must have i) that $\hat{e}>e_{1}=\frac{\beta_{2}}{\beta_{0}-\beta_{3}}=\frac{\frac{\alpha \sigma^{2}}{2}-\hat{e} \beta_{3}}{\beta_{0}-\beta_{3}}$, which is equivalent to $\beta_{0}>\frac{1}{\hat{e}} \frac{\alpha \sigma^{2}}{2}$ and ii) $e_{2}>\hat{e}$. $e_{2}$ solves $\frac{\alpha \sigma^{2}}{2}-\hat{e} \beta_{3}+\beta_{3} e_{2}=-\beta_{3} \ln \left(1-e_{2}\right)$. One observes that $e_{2}$ is decreasing in $\beta_{3}$ and that $\lim _{\beta_{3} \rightarrow 0} e_{2}=1$. Hence, for each value of $\hat{e}$ one can take $\beta_{0}$ large enough, $\beta_{3}$ small enough, and take $\beta_{2}=\frac{\alpha \sigma^{2}}{2}-\hat{e} \beta_{3}$ so that $\hat{e} \in\left[e_{1}, e_{2}\right]$.

Finally, if $\hat{e} \in\left[e_{1}, e_{2}\right]$ and $\beta_{2}=\frac{\alpha \sigma^{2}}{2}-\hat{e} \beta_{3}$, then (13) can be written as

$$
\frac{\frac{\alpha \sigma^{2}}{2}-\hat{e} \beta_{3}+\hat{e} \beta_{3}}{\beta_{3}}>(1+\alpha)(1-\hat{e})
$$

which is satisfied for all $\beta_{3}<\frac{\alpha}{1+\alpha} \frac{\sigma^{2}}{2} \frac{1}{1-\hat{e}}$

Proof of Proposition 5. Let $y(e)=\frac{g_{e}(e)}{g_{e e}(e)}-(1+\alpha)(1-e)$. We note that $\lim _{e \rightarrow 1} y(e)=0$ : since $\lim _{e \rightarrow 1} g_{e}(e)=\infty$ and $g_{e e}(e)>0 \forall e, g_{e}(e)$ must be convex for $e$ close to 1 ; hence, for $e$ close to $1 g_{e e}(e) \geq e g_{e e}(e)>g_{e}(e)$; hence $\lim _{e \rightarrow 1} \frac{g_{e}(e)}{g_{e e}(e)}=0$ and hence $\lim _{e \rightarrow 1} y(e)=0$. Note further that $\left.y(e)\right|_{e=0}=-(1+\alpha)$, which follows from $g_{e e}(e)>0 \forall e$. Using the multiplicative form we observe that $y(e)=\frac{h_{e}(e)}{h_{e e}(e)}-(1+\alpha)(1-e)$ so that our assumptions imply $y(e)$ concave in $e$. Differentiating $y$ with respect to $e$ one obtains $\frac{\partial y(e)}{\partial e}=(2+\alpha)-\frac{h_{e}(e) h_{e e e}(e)}{\left(h_{e e}(e)\right)^{2}}$. 
Hence $\frac{\partial y\left(e\left(\Delta, \frac{\alpha}{\beta}\right)\right)}{\partial \beta}=\left((2+\alpha)-\frac{h_{e}\left(e\left(\Delta, \frac{\alpha}{\beta}\right)\right) h_{e e e}\left(e\left(\Delta, \frac{\alpha}{\beta}\right)\right)}{\left(h_{e e}\left(e\left(\Delta, \frac{\alpha}{\beta}\right)\right)\right)^{2}}\right) \frac{\partial e\left(\Delta, \frac{\alpha}{\beta}\right)}{\partial \beta}$. Since $\lim _{\beta \rightarrow 0} e\left(\Delta, \frac{\alpha}{\beta}\right)=1$ and $\frac{\partial e\left(\Delta, \frac{\alpha}{\beta}\right)}{\partial \beta}<0 \forall \beta, \lim _{e \rightarrow 1} \frac{h_{e}(e) h_{e e e}(e)}{\left(h_{e e}(e)\right)^{2}}>2+\alpha$ implies that $\lim _{\beta \rightarrow 0} \frac{\partial y\left(e\left(\Delta, \frac{\alpha}{\beta}\right)\right)}{\partial \beta}>0$. Hence $y\left(e\left(\Delta, \frac{\alpha}{\beta}\right)\right)>$ 0 for $\beta$ positive but small. Since $y(e)$ is concave in $e$ and $e\left(\Delta, \frac{\alpha}{\beta}\right)$ monotonic in $\beta$, there exists a unique value of $\beta, \beta^{\prime}$, such that

$$
\left.\frac{h_{e}\left(e\left(\Delta, \frac{\alpha}{\beta^{\prime}}\right)\right)}{h_{e e}\left(e\left(\Delta, \frac{\alpha}{\beta^{\prime}}\right)\right)}\right|_{\Delta=0}=\left.(1+\alpha)\left(1-e\left(\Delta, \frac{\alpha}{\beta^{\prime}}\right)\right)\right|_{\Delta=0}
$$

Hence (13) is satisfied iff $\beta \in\left(0, \beta^{\prime}\right)$.

Proof of Proposition 6. $\quad \underline{\Delta}$ the first and second order condition for an optimum, i.e. $\left.P_{\Delta}\left(\Delta, \alpha, \frac{\alpha}{\beta}\right)\right|_{\Delta=\underline{\Delta}}=0$ and $\left.P_{\Delta \Delta}\left(\Delta, \alpha, \frac{\alpha}{\beta}\right)\right|_{\Delta=\underline{\Delta}}<0$. In what follows we use notation $P_{\Delta}\left(\underline{\Delta}, \alpha, \frac{\alpha}{\beta}\right)=$ $\left.P_{\Delta}\left(\Delta, \alpha, \frac{\alpha}{\beta}\right)\right|_{\Delta=\underline{\Delta}}$. By the implicit function theorem

$$
\frac{\partial \underline{\Delta}}{\partial \beta}=\frac{P_{\Delta \beta}\left(\underline{\Delta}, \alpha, \frac{\alpha}{\beta}\right)}{-P_{\Delta \Delta}\left(\underline{\Delta}, \alpha, \frac{\alpha}{\beta}\right)}
$$

Clearly, $-P_{\Delta \Delta}\left(\underline{\Delta}, \alpha, \frac{\alpha}{\beta}\right)>0$.

$$
P_{\Delta \beta}\left(\underline{\Delta}, \alpha, \frac{\alpha}{\beta}\right) \propto \frac{\partial}{\partial e}\left((1+\alpha) e\left(\underline{\Delta}, \frac{\alpha}{\beta}\right)+\frac{h_{e}\left(e\left(\underline{\Delta}, \frac{\alpha}{\beta}\right)\right)}{h_{e e}\left(e\left(\underline{\Delta}, \frac{\alpha}{\beta}\right)\right)}\right) \frac{\partial e\left(\underline{\Delta}, \frac{\alpha}{\beta}\right)}{\partial \beta}
$$

Since $(1+\alpha) e+\frac{h_{e}(e)}{h_{e e}(e)}$ is concave in $e,(1+\alpha) e+\frac{h_{e}(e)}{h_{e e}(e)}$ is maximized for $e^{\prime \prime}$ that satisfies $2+\alpha=$ $\left.\frac{h_{e}(e) h_{e e e}(e)}{\left(h_{e e}(e)\right)^{2}}\right|_{e=e^{\prime \prime}}$ and therefore $\frac{\partial}{\partial e}\left((1+\alpha) e+\frac{h_{e}(e)}{h_{e e}(e)}\right) \lessgtr \Leftrightarrow e \gtrless e^{\prime \prime}$. Thus, we need to show that $\frac{d e\left(\underline{\Delta}, \frac{\alpha}{\beta}\right)}{d \beta}<0$. By straightforward differentiation of (11) and some manipulations, using (26), one obtains

$$
\begin{gathered}
\frac{d e}{d \beta}=\frac{\partial e\left(\underline{\Delta}, \frac{\alpha}{\beta}\right)}{\partial \beta}+\frac{\partial e\left(\underline{\Delta}, \frac{\alpha}{\beta}\right)}{\partial \Delta} \frac{\partial \underline{\Delta}}{\partial \beta}<0 \\
\Leftrightarrow \\
1-\left(e\left(\underline{\Delta}, \frac{\alpha}{\beta}\right)+\frac{g_{e}\left(e\left(\underline{\Delta}, \frac{\alpha}{\beta}\right)\right)}{g_{e e}\left(e\left(\underline{\Delta}, \frac{\alpha}{\beta}\right)\right)}\right)\left(E \pi_{\Delta}(\Delta, \phi)-E \pi_{\Delta}(\Delta, \eta)\right)>0 \\
\Leftrightarrow \\
1-\frac{\Delta\left(E \pi_{\Delta \Delta}(\Delta, \phi)-E \pi_{\Delta \Delta}(\Delta, \eta)\right)}{E \pi_{\Delta}(\Delta, \phi)-E \pi_{\Delta}(\Delta, \eta)}>0
\end{gathered}
$$

The last equivalence follows from using $\left.P_{\Delta}\left(\Delta, \alpha, \frac{\alpha}{\beta}\right)\right|_{\Delta=\underline{\Delta}}=0$ to substitute $\frac{\Delta}{E \pi_{\Delta}(\Delta, \phi)-E \pi_{\Delta}(\Delta, \eta)}$ for $e\left(\underline{\Delta}, \frac{\alpha}{\beta}\right)+\frac{g_{e}\left(e\left(\underline{\Delta}, \frac{\alpha}{\beta}\right)\right)}{g_{e e}\left(e\left(\underline{\underline{\alpha}}, \frac{\alpha}{\beta}\right)\right)}$. Since $\left.\left(E \pi_{\Delta}(\Delta, \phi)-E \pi_{\Delta}(\Delta, \eta)\right)\right|_{\Delta=0}$ and $\left(E \pi_{\Delta}(\Delta, \phi)-E \pi_{\Delta}(\Delta, \eta)\right)$ is increasing concave for all $\Delta>0$, the result follows. Therefore there is a unique $\beta^{\prime \prime}$ associated to 
$e^{\prime \prime}$ by the equation $e^{\prime \prime}=e\left(\underline{\Delta}, \frac{\alpha}{\beta^{\prime \prime}}\right)$.

For $\beta$ small enough $\underline{\Delta}=\Delta^{*}$. To see this, consider (25):

$$
\begin{aligned}
P_{\Delta \Delta}(\Delta, \alpha)= & \underbrace{-(1+\alpha)+\left(e(\cdot)(1+\alpha)+\frac{g_{e}(e(\cdot))}{g_{e e}(e(\cdot))}\right)\left(E \pi_{\Delta \Delta}(\Delta, \phi)-E \pi_{\Delta \Delta}(\Delta, \eta)\right)}_{=X(\Delta, \beta)} \\
& +\underbrace{\frac{\partial}{\partial e}\left(e(\cdot)(1+\alpha)+\frac{g_{e}(e(\cdot))}{g_{e e}(e(\cdot))}\right) \frac{\alpha\left(E \pi_{\Delta}(\Delta, \phi)-E \pi_{\Delta}(\Delta, \eta)\right)^{2}}{g_{e e}(e(\cdot))}}_{=Y(\Delta, \beta)}
\end{aligned}
$$

Since the agent's effort is increasing in $\Delta$ for all $\Delta$ it follows that $Y(\Delta, \beta) \leq 0$ for all $\Delta \geq \Delta$ if $\beta \leq$ $\beta^{\prime \prime}$. Consider next $X(\Delta, \beta)$. Using the first order condition, $\left.P_{\Delta}\left(\Delta, \alpha, \frac{\alpha}{\beta}\right)\right|_{\Delta=\underline{\Delta}}=0$, and the fact that $E \pi_{\Delta}(\Delta, \phi)-E \pi_{\Delta}(\Delta, \eta)$ is increasing concave in $\Delta$ and satisfies $\left.\left(E \pi_{\Delta}(\Delta, \phi)-E \pi_{\Delta}(\Delta, \eta)\right)\right|_{\Delta=0}=$ 0 we observe that $X(\underline{\Delta}, \beta)<0 \forall \beta$. Moreover, $X(\Delta, \beta)$ is decreasing in $\Delta$ for $\Delta \geq \underline{\Delta}$ if $\beta \leq \beta^{\prime \prime}$, because $\frac{\partial}{\partial e}\left(e(\cdot)(1+\alpha)+\frac{g_{e}(e(\cdot))}{g_{e e}(e(\cdot))}\right) \leq 0$ for $e \geq e^{\prime \prime}$ and $E \pi_{\Delta}(\Delta, \phi)-E \pi_{\Delta}(\Delta, \eta)$ is increasing concave in $\Delta$.

Proof of Proposition 7. The proof follows from straightforward extensions of the proofs to propositions 3 and 5 . In this case

$$
P(\Delta, \alpha)=E V(x(\phi), \tilde{\eta})+\frac{e}{\alpha} g_{e}(e)
$$

and

$$
P_{\Delta}(\Delta, \alpha)=\left(e(\cdot)+\frac{g_{e}(e(\cdot))}{g_{e e}(e(\cdot))}\right)\left(E \pi_{\Delta}(\Delta, \phi)-E \pi_{\Delta}(\Delta, \eta)\right)-\Delta
$$

From $\left.P_{\Delta \Delta}(\Delta, \alpha)\right|_{\Delta=0}$ one finds that $y(e)=\frac{g_{e}(e)}{g_{e e}(e)}-(1-e)$. If $\lim _{e \rightarrow 1} \frac{g_{e}(e) g_{e e e}(e)}{\left(g_{e e}(e)\right)^{2}}>2$ then $\lim _{e \rightarrow 1} \frac{\partial y(e)}{\partial e}<0$. The remainder of the proof is a trivial extension of the proof to proposition 5.

Proof of Proposition 8. Define $S(\Delta, \alpha)=e(\Delta, \alpha)+\frac{1}{1+\alpha} \frac{g_{e}(e(\Delta, \alpha))}{g_{e e}(e(\Delta, \alpha))}$ and let $S(0, \alpha)=$ $\left.\left(e(\Delta, \alpha)+\frac{1}{1+\alpha} \frac{g_{e}(e(\Delta, \alpha))}{g_{e e}(e(\Delta, \alpha))}\right)\right|_{\Delta=0}$. (13) is satisfied iff $S(0, \alpha)>1$. Using $e_{\alpha}(\Delta, \alpha)=\frac{1}{\alpha} \frac{g_{e}(e(\Delta, \alpha))}{g_{e e}(e(\Delta, \alpha))}$ one finds

$$
\begin{aligned}
S_{\alpha}(\Delta, \alpha) & =\frac{\partial}{\partial \alpha}\left(e(\Delta, \alpha)+\frac{1}{1+\alpha} \frac{g_{e}(e(\Delta, \alpha))}{g_{e e}(e(\Delta, \alpha))}\right) \\
& =\left(\frac{g_{e}(e(\Delta, \alpha))}{g_{e e}(e(\Delta, \alpha))}\left(\frac{1}{\alpha}-\frac{1}{(1+\alpha)^{2}}\right)+\frac{1}{1+\alpha} \frac{\partial}{\partial e}\left(\frac{g_{e}(e(\Delta, \alpha))}{g_{e e}(e(\Delta, \alpha))}\right) e_{\alpha}(\Delta, \alpha)\right)
\end{aligned}
$$

Clearly, our assumptions imply $S_{\alpha}(\Delta, \alpha)>0$ for all $\Delta$. Since $\left.\left(g_{e}(e)\right)\right|_{e=1}<\left.\infty(e(\Delta, \alpha))\right|_{\Delta=0}$ tends to 1 as $\alpha$ increases. By continuity there must be some $\alpha$ such that $\left.(e(\Delta, \alpha))\right|_{\Delta=0}<1$ and $S(0, \alpha)>1$. By monotonicity of $S(0, \alpha)$ there is a unique $\alpha^{\prime}$ such that $S\left(0, \alpha^{\prime}\right)=1$ and $(13)$ is 
satified for $\alpha>\alpha^{\prime}$.

From (15) with $\beta \equiv 1, \underline{\Delta}$ satisfies, for interior solutions,

$$
\left(\left(e(\Delta, \alpha)+\frac{1}{1+\alpha} \frac{g_{e}(e(\Delta, \alpha))}{g_{e e}(e(\Delta, \alpha))}\right)\left(E \pi_{\Delta}(\Delta, \phi)-E \pi_{\Delta}(\Delta, \eta)\right)\right)-\left.\Delta\right|_{\Delta=\underline{\Delta}}=0
$$

Hence, by Milgrom and Roberts' (1990) theorem 1, the smallest interior solution is nondecreasing in $\alpha$. It is strictly increasing if $\underline{\Delta}>0$. By the arguments above $\underline{\Delta}>0$ over a nonempty range. By monotonicity this range must be a convex set. Since $e(\underline{\Delta}, \alpha)$ is increasing in $\alpha$, the contract eventually implements $e(\underline{\Delta}, \alpha)=1$. Define $\alpha^{\prime \prime}=\min \{\alpha: e(\underline{\Delta}, \alpha)=1\}$. For $\alpha \geq \alpha^{\prime \prime}$, the optimal solution is a corner solution and $\underline{\Delta}$ is the smallest restriction that implements $e(\underline{\Delta}, \alpha)=1$. Since $e(\Delta, \alpha)$ is nondecreasing in $\alpha$, and strictly increasing in $\alpha$ if $e(\Delta, \alpha)<1$ the principal optimally decreases $\underline{\Delta}$ to keep $e(\underline{\Delta}, \alpha)$ constant at 1 .

\section{Appendix $\mathbf{B}^{19}$}

Lemma B. Problem (16) s.t. (17), (18), (19), and (20)is solved if and only if the following problem is solved optimally:

$$
\begin{gathered}
\max _{\substack{x(\eta) \\
x(\phi), e}}(1+\alpha) K+e\left\{\int_{\underline{\eta}}^{\bar{\eta}}\left(-\frac{\alpha+1}{2}(x(\eta)-\eta)^{2}\right) d F(\eta)\right\}-(1-e) \frac{\alpha+1}{2}\left((x(\phi)-\mu)^{2}+\sigma^{2}\right) \\
-g(e)+\lambda\left\{\int_{\underline{\eta}}^{\bar{\eta}} \alpha(x(\eta)-\eta)\left(1_{\eta>\mu}-F(\eta)\right) d \eta+\frac{\alpha \sigma^{2}}{2}-g_{e}(e)\right\} \\
\text { s.t. } \frac{\partial x(\eta)}{\partial \eta} \geq 0 ; x(\mu)=x(\phi)
\end{gathered}
$$

The proof uses standard arguments. The reader is referred to Szalay (2001). Here, we prove only the pooling property. Taking (17) and (18) at $\eta=\mu$ and rearranging the constraints yields

$$
\int \frac{\alpha}{2}(x(\mu)-\eta)^{2} d F(\eta)-\int \frac{\alpha}{2}(x(\phi)-\eta)^{2} d F(\eta) \geq \tau(\mu)-\tau(\phi)
$$

and

$$
\tau(\mu)-\tau(\phi) \geq \frac{\alpha}{2}(x(\mu)-\mu)^{2}-\frac{\alpha}{2}(x(\phi)-\mu)^{2}
$$

\footnotetext{
${ }^{19}$ The development of the solution concept builds on Lewis and Sappington (1993) and Crémer, Khalil and Rochet (1998). Both, Lewis and Sappingtion and Crémer et.al. study modified versions of the procurement problem originally due to Baron and Myerson. In Lewis and Sappington the adverse selection problem is complicated by the possibility of ignorance by the agent. The probability of ignorance is taken as exogenous in their model. In Crémer et.al information acquisition is endogenous. Information acquisition involves a discrete cost and $e \in\{0,1\}$ in their model so that the agent is in equilibrium either completely informed or completely ignorant. In the present analysis the adverse selection problem is complicated by the simultaneous presence of both problems.
} 
Both inequalities can hold simultaneously only if $\tau(\mu)=\tau(\phi)$. But then also $x(\mu)=x(\phi)$.

Proof of Proposition 9. Problem (M) is solved by a three step sequential maximization procedure. In step 1 we solve for a constrained optimal function $x(\eta \mid \bar{x}, \bar{e})$ taking as given that the principal implements some $x(\phi)=\bar{x}$ and $e=\bar{e}$. In step 2, we take account of the optimal solution to step 1 and treat $x(\phi)$ as a choice variable, obtaining this way a constrained function $x(\eta \mid x(\phi), \bar{e})$ and an optimal $x(\phi \mid \bar{e})$. In the final third step $e$ is endogenized which delivers the optimal function $x(\eta)$ and the optimal effort level the principal implements.

Step 1. Abstract from the constraint $x(\phi)=\bar{x}$ for a second. The standard way to solve this problem is to neglect the monotonicity constraint and derive the optimal solution by pointwise maximization. Afterwards one imposes conditions on $F(\cdot)$ so that the pointwise optimum is monotonic in $\eta$. Anticipating the solution we assume that $f(\eta)$ is nondecreasing for $\eta \leq \mu$ and non-increasing for $\eta>\mu$.

Consider now the role of the constraint $x(\phi)=\bar{x}$. Under our assumptions on hazard rates the solution to step 1 will be monotonic if not the constraint $x(\phi)=\bar{x}$ introduces a nonmonoticity. Distinguish two cases: $i$ ) $\bar{x} \geq \mu$ and $i i) \bar{x}<\mu$.

Case $i$ ) : The maximization problem is:

$$
\begin{aligned}
& \max _{x(\eta)}(1+\alpha) K+e\left\{\int_{\underline{\eta}}^{\bar{\eta}}\left(-\frac{\alpha+1}{2}(x(\eta)-\eta)^{2}\right) d F(\eta)\right\}-(1-e) \frac{\alpha+1}{2}\left((\bar{x}-\mu)^{2}+\sigma^{2}\right)-g(e) \\
& \quad+\lambda(\bar{x}, \bar{e})\left\{\int_{\underline{\eta}}^{\bar{\eta}} \alpha(x(\eta)-\eta)\left(1_{\eta>\mu}-F(\eta)\right) d \eta+\frac{\alpha \sigma^{2}}{2}-g_{e}(\bar{e})\right\}+1_{\eta \geq \mu} \kappa(\eta)\{x(\eta)-\bar{x}\}
\end{aligned}
$$

In this problem, $\kappa(\eta)$ is, for each value of $\eta \geq \mu$, the Kuhn-Tucker multiplier one the constraint $x(\eta) \geq \bar{x}$. The Lagrange multiplier, $\lambda(\bar{x}, \bar{e})$, is a function of the imposed parameters. By pointwise maximization one finds from the first order conditions of (M1):

$$
\begin{aligned}
& x(\eta \mid \bar{x}, \bar{e})=\eta-\frac{\lambda(\bar{x}, \bar{e})}{\bar{e}} \frac{\alpha}{1+\alpha} \frac{F(\eta)}{f(\eta)} \text { for } \eta<\mu \\
& x(\eta \mid \bar{x}, \bar{e})=\max \left\{\bar{x}, \eta+\frac{\lambda(\bar{x}, \bar{e})}{\bar{e}} \frac{\alpha}{1+\alpha} \frac{1-F(\eta)}{f(\eta)}\right\} \text { for } \eta \geq \mu .
\end{aligned}
$$

$\frac{\lambda(\bar{x}, \bar{e})}{\bar{e}}$ is uniquely defined by the incentive constraint:

$$
\frac{\lambda(\bar{x}, \bar{e})}{\bar{e}}=\frac{g_{e}(\bar{e})-\frac{\alpha \sigma^{2}}{2}}{\int_{\underline{\eta}}^{\mu} \frac{\alpha^{2}}{1+\alpha} \frac{F(\eta)^{2}}{f(\eta)} d \eta+\int_{\check{\eta}(\bar{x})}^{\bar{\eta}} \frac{\alpha^{2}}{1+\alpha} \frac{(1-F(\eta))^{2}}{f(\eta)} d \eta+\int_{\mu}^{\check{\eta}(\bar{x})} \alpha(\bar{x}-\eta)(1-F(\eta)) d \eta}
$$

In this expression, $\check{\eta}(\bar{x})$ is the value of $\eta$ such that $\bar{x}=\check{\eta}(\bar{x}, \bar{e})+\frac{\lambda(\bar{x}, \bar{e})}{\bar{e}} \frac{\alpha}{1+\alpha} \frac{1-F(\check{\eta}(\bar{x}, \bar{e}))}{f(\check{\eta}(\bar{x}, \bar{e}))}$. Assume for the moment that $\frac{\lambda(\bar{x}, \bar{e})}{\bar{e}} \frac{\alpha}{1+\alpha}<1$. Then, our assumptions on the density imply that the pointwise 
optimum is monotonic in $\eta$. We show in step 3 that $\frac{\lambda(\bar{x}, \bar{e})}{\bar{e}} \frac{\alpha}{1+\alpha}<1$ is true.

Step 2: Substitute (28) into (M1) and replace the parameter $\bar{x}$ by the choice variable $x(\phi)$. The objective function is

$$
\begin{aligned}
& P(x(\phi), \bar{e}, \cdot)=\bar{e}\left\{\begin{array}{c}
\int_{\underline{\eta}}^{\mu}\left(-\frac{1+\alpha}{2}\right)\left(\frac{\lambda(x(\phi), \bar{e})}{\bar{e}} \frac{\alpha}{1+\alpha} \frac{F(\eta)}{f(\eta)}\right)^{2} d F(\eta)-\int_{\mu}^{\grave{\eta}(x(\phi, \bar{e}))}\left(\frac{1+\alpha}{2}\right)(x(\phi)-\eta)^{2} d F(\eta) \\
-\int_{\check{\eta}(x(\phi), \bar{e})}^{\bar{\eta}}\left(\frac{1+\alpha}{2}\right)\left(\frac{\lambda(x(\phi), \bar{e})}{\bar{e}} \frac{\alpha}{1+\alpha} \frac{(1-F(\eta))}{f(\eta)}\right)^{2} d F(\eta)
\end{array}\right\} \\
& -(1-\bar{e}) \frac{\alpha+1}{2}(x(\phi)-\mu)^{2}+(1+\alpha) K-g(\bar{e}) \\
& +\lambda(x(\phi), \bar{e})\left\{\int_{\underline{\eta}}^{\bar{\eta}} \alpha(x(\eta \mid x(\phi), \bar{e})-\eta)\left(1_{\eta>\mu}-F(\eta)\right) d \eta+\frac{\alpha \sigma^{2}}{2}-g_{e}(\bar{e})\right\} .
\end{aligned}
$$

and the maximization problem is

$$
\max _{x(\phi)} P(x(\phi), \bar{e}, \cdot)
$$

By straightforward calculus

$$
\begin{aligned}
& \frac{\partial P(x(\phi), \bar{e}, \cdot)}{\partial x(\phi)} \\
= & \bar{e}\left\{\begin{array}{c}
\int_{\underline{\eta}}^{\mu} \frac{-\alpha^{2}}{1+\alpha} \frac{F(\eta)^{2}}{f(\eta)}\left(\frac{\lambda(x(\phi), \bar{e})}{\bar{e}}\right) d \eta \frac{\partial\left(\frac{\lambda(x(\phi), \bar{e})}{\bar{e}}\right)}{\partial x(\phi)}-\int_{\mu}^{\check{\eta}(x(\phi, \bar{e}))}(\alpha+1)(x(\phi)-\eta) d F(\eta) \\
-\int_{\breve{\eta}(x(\phi, \bar{e}))}^{\bar{\eta}} \frac{\alpha^{2}}{1+\alpha} \frac{(1-F(\eta))^{2}}{f(\eta)}\left(\frac{\lambda(x(\phi), \bar{e})}{\bar{e}}\right) d \eta \frac{\partial\left(\frac{\lambda(x(\phi), \bar{e})}{\bar{e}}\right)}{\partial x(\phi)}
\end{array}\right\} \\
- & (1-\bar{e})(1+\alpha)(x(\phi)-\mu) .
\end{aligned}
$$

To get this expression we used the fact that - by definition - the incentive constraint holds with equality, i.e. that

$$
\lambda(x(\phi), \bar{e})\left\{\int_{\underline{\eta}}^{\bar{\eta}} \alpha(x(\eta \mid x(\phi), \bar{e})-\eta)\left(1_{\eta>\mu}-F(\eta)\right) d \eta+\frac{\alpha \sigma^{2}}{2}-g_{e}(\bar{e})\right\} \equiv 0 \forall x(\phi)
$$

Totally differentiating this identity and using (28) to substitute for $x(\phi)$ one obtains an expression that can be used to simplify $\frac{\partial P(x(\phi), \bar{e}, \cdot)}{\partial x(\phi)}$. More specifically, one finds that

$$
\begin{aligned}
\frac{\partial P(x(\phi), \bar{e}, \cdot)}{\partial x(\phi)}= & \bar{e}\left\{\int_{\mu}^{\check{\eta}(x(\phi, \bar{e}))} \frac{\lambda(x(\phi), \bar{e})}{\bar{e}} \alpha(1-F(\eta))-(1+\alpha)(x(\phi)-\eta) f(\eta) d \eta\right\} \\
& -(1-\bar{e})(1+\alpha)
\end{aligned}
$$

By definition of $\check{\eta}(x(\phi, \bar{e}))$ we have $x(\phi)>\eta+\frac{\lambda(x(\phi), \bar{e})}{\bar{e}} \frac{\alpha}{1+\alpha} \frac{1-F(\eta)}{f(\eta)}$ for all $\eta \in[\mu, \check{\eta}(x(\phi))]$ so that this expression is strictly negative. Hence the solution to step 2 is

$$
\begin{aligned}
& x(\eta \mid \bar{e})=\eta-\frac{\lambda(\bar{e})}{\bar{e}} \frac{\alpha}{1+\alpha} \frac{F(\eta)}{f(\eta)} \text { for } \eta<\mu \\
& x(\phi \mid \bar{e})=\mu \quad \text { for } \eta=\mu \\
& x(\eta \mid \bar{e})=\eta+\frac{\lambda(\bar{e})}{\bar{e}} \frac{\alpha}{1+\alpha} \frac{1-F(\eta)}{f(\eta)} \text { for } \eta>\mu .
\end{aligned}
$$


Step 3: Substitute (31) into the (M2) and replace the parameter $\bar{e}$ by the choice variable $e$. The objective is now

$$
\begin{aligned}
P(e, \cdot)= & (1+\alpha) K-(1-e)\left(\frac{\alpha+1}{2}\right) \sigma^{2} \\
& -e\left\{\int_{\underline{\eta}}^{\bar{\eta}} \frac{\alpha+1}{2}\left(\frac{\alpha}{\alpha+1} \frac{\lambda(\bar{e})}{\bar{e}} \frac{1_{\eta>\mu}-F(\eta)}{f(\eta)}\right)^{2} d F(\eta)\right\}-g(e) \\
& +\lambda(e)\left\{\int_{\underline{\eta}}^{\bar{\eta}} \alpha(x(\eta \mid e)-\eta)\left(1_{\eta>\mu}-F(\eta)\right) d \eta+\frac{\alpha \sigma^{2}}{2}-g_{e}(e)\right\}
\end{aligned}
$$

The maximization problem is $\max _{e} P(e, \cdot)$. By straightforward calculus

$$
\begin{aligned}
\frac{\partial P(e, \cdot)}{\partial e}=\frac{\alpha+1}{2} \sigma^{2}-\int_{\underline{\eta}}^{\bar{\eta}}( & \left.\frac{\alpha^{2}}{2(1+\alpha)}\left(\frac{\lambda(e)}{e} \frac{1_{\eta>\mu}-F(\eta)}{f(\eta)}\right)^{2}\right) d F(\eta)-g_{e}(e) \\
& -e\left\{\int_{\underline{\eta}}^{\bar{\eta}} \frac{\alpha^{2}}{2(1+\alpha)}\left(\frac{1_{\eta>\mu}-F(\eta)}{f(\eta)}\right)^{2} d F(\eta)\right\} \frac{2 \lambda(e)}{e} \frac{\partial}{\partial e}\left[\frac{\beta(e)}{e}\right]
\end{aligned}
$$

To find this expression we use again the fact that by definition the incentive constraint holds with equality for all $e$, i.e.,

$$
\lambda(e)\left\{\int_{\underline{\eta}}^{\bar{\eta}} \alpha(x(\eta)-\eta)\left(1_{\eta>\mu}-F(\eta)\right) d \eta+\frac{\alpha \sigma^{2}}{2}-g_{e}(e)\right\} \equiv 0 \forall e
$$

Using (31) to substitute for $x(\eta)$, this identity implies that

$$
\frac{\lambda(e)}{e}=\frac{g_{e}(e)-\frac{\alpha \sigma^{2}}{2}}{\int_{\underline{\eta}}^{\bar{\eta}}\left(\frac{\alpha^{2}}{1+\alpha} \frac{\left(1_{\eta>\mu}-F(\eta)\right)^{2}}{f(\eta)}\right) d \eta}
$$

Letting $\hat{e}=g_{e}^{-1}\left(\frac{\alpha \sigma^{2}}{2}\right)$ we find that $\left.\left(\frac{\lambda(e)}{e}\right)\right|_{e=\hat{e}}=0$. Hence

$$
\left.\frac{\partial P(e, \cdot)}{\partial e}\right|_{e=\hat{e}}=\frac{\alpha+1}{2} \sigma^{2}-\left.g_{e}(e)\right|_{e=\hat{e}}=\frac{\sigma^{2}}{2}>0
$$

so that it is optimal to introduce ex post distortions. The optimal effort level satisfies the first order condition

$$
\left.\left(\frac{\alpha+1}{2} \sigma^{2}-g_{e}(e)-\frac{\frac{1+\alpha}{2}\left(\frac{g_{e}(e)}{\alpha}-\frac{\sigma^{2}}{2}\right)^{2}}{\int_{\underline{\eta}}^{\bar{\eta}}\left(\frac{\left(1_{\eta>\mu}-F(\eta)\right)^{2}}{f(\eta)}\right) d \eta}-e \frac{\frac{1+\alpha}{\alpha}\left(\frac{g_{e}(e)}{\alpha}-\frac{\sigma^{2}}{2}\right)}{\int_{\underline{\eta}}^{\bar{\eta}}\left(\frac{\left(1_{\eta>\mu}-F(\eta)\right)^{2}}{f(\eta)}\right) d \eta} g_{e e}(e)\right)\right|_{e=e *}=0
$$

and the second order condition

$$
\left.\left(-g_{e e}(e)-\frac{\frac{1+\alpha}{\alpha}\left(\frac{g_{e}(e)}{\alpha}-\frac{\sigma^{2}}{2}\right)}{\int_{\underline{\eta}}^{\bar{\eta}}\left(\frac{\left(1_{\eta>\mu}-F(\eta)\right)^{2}}{f(\eta)}\right) d \eta}\left(2 g_{e e}(e)+e \frac{g_{e e}(e)^{2}}{\alpha}+e g_{e e e}(e)\right)\right)\right|_{e=e *}<0
$$


So that $g_{e e e}(e)>0$ implies that the first order condition is sufficient for a local optimum. From the first order condition we derive

$$
\left.\left(\frac{\left(\frac{g_{e}(e)}{\alpha}-\frac{\sigma^{2}}{2}\right)}{\int_{\underline{\eta}}^{\bar{\eta}}\left(\frac{\left(1_{\eta>\mu}-F(\eta)\right)^{2}}{f(\eta)}\right) d \eta}\right)\right|_{e=e *}=\left.\left(\frac{\frac{\alpha+1}{2} \sigma^{2}-g_{e}(e)}{\frac{1+\alpha}{2}\left(\frac{g_{e}(e)}{\alpha}-\frac{\sigma^{2}}{2}\right)+e \frac{1+\alpha}{\alpha} g_{e e}(e)}\right)\right|_{e=e *}
$$

If $g_{\text {eee }}(e) \geq 0$ then $e \frac{1+\alpha}{\alpha} g_{e e}(e) \geq \frac{1+\alpha}{\alpha} g_{e}(e)>\frac{\alpha+1}{2} \sigma^{2}$ so that the right hand side is smaller than one.

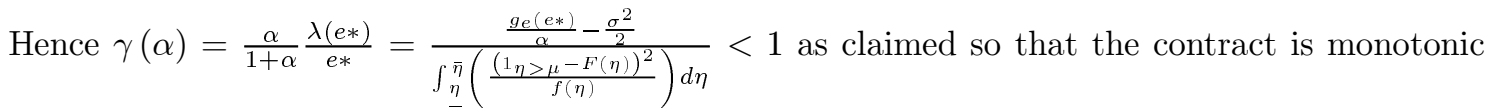
and the pointwise optimum is the optimal contract.

Finally, for $\alpha$ large the effort level is the unique globally optimal effort level. $\frac{\left(\frac{g_{e}(e)}{\alpha}-\frac{\sigma^{2}}{2}\right)}{\int_{\underline{\eta}}^{\bar{\eta}}\left(\frac{\left(1_{\eta}>\mu-F(\eta)\right)^{2}}{f(\eta)}\right) d \eta} \leq$ $\frac{\left(\frac{\alpha+1}{2 \alpha} \sigma^{2}-\frac{\sigma^{2}}{2}\right)}{\int_{\underline{\eta}}^{\eta}\left(\frac{\left(1_{\eta}>\mu-F(\eta)\right)^{2}}{f(\eta)}\right) d \eta}=\frac{\sigma^{2}}{2 \alpha \int_{\underline{\eta}}^{\eta}\left(\frac{\left(1_{\eta}>\mu-F(\eta)\right)^{2}}{f(\eta)}\right) d \eta}$. Hence, for $\alpha \geq \frac{\sigma^{2}}{2 \int_{\underline{\eta}}^{\eta}\left(\frac{\left(1_{\eta}>\mu-F(\eta)\right)^{2}}{f(\eta)}\right) d \eta} \gamma(\alpha)$ is bounded above by 1 so that the principal's problem is concave in $e$ for any $e$.

\section{$9 \quad$ References}

- Aghion, P. and Tirole, J. (1997) "Formal and Real Authority" Journal of Political Economy $105(1), 1-29$

- Armstrong, Mark (1994) "Delegation and Discretion" Discussion Papers in Economics and Econometrics No. 9421, University of Southampton

- Baron, D. and Myerson, R. (1982) "Regulating a Monopolist with Unknown Costs" Econometrica 50(4), 911-930

- Bergemann, D. and Välimäki, J. (2002) "Information Acquisition and Efficient Mechanism Design" forthcoming Econometrica

- Blackwell, D. (1950) "Comparison of Experiments", reprinted in Blackwell, D. and Girshick, M. (1954) "Theory of Games and Statistical Decisions", John WileyESSons, New York

- Crawford, V. and Sobel, J. (1982) "Strategic Information Transmission" Econometrica 50(6), $1431-1451$

- Crémer, J. and Khalil, F. (1992) "Gathering Information before Signing a Contract" American Economic Review, 82(3), 566-578

- Crémer, J., Khalil, F. and Rochet, J-C. (1998a)" Contracts and Productive Information Gathering" Games and Economic Behavior 25, 174-193 
- Crémer, J., Khalil, F. and Rochet, J.-C. (1998b) "Strategic Information Gathering before a Contract is Offered" Journal of Economic Theory 81, 163-200

- Crémer, J. and McLean (1988) "Full Extraction of the Surplus in Bayesian and Dominant Strategy Auctions" Econometrica 56, 1247-57

- Demski, J. and Sappingtion, D. (1987) "Delegated Expertise" Journal of Accounting Research $\mathbf{2 5}(1), 68-89$

- Dessein, W. (2002) "Authority and Communication in Organizations" forthcoming Review of Economic Studies

- de Garidel-Thoron, T. and Ottaviani, M. (2000) "The Economics of Advice" mimeo UCL

- deGroot, M. (1970) "Optimal Statistical Decisions" McGraw-Hill, New York

- Dewatripont, M. and Tirole, J. (1999) "Advocates" Journal of Political Economy 107(1), $1-39$

- Green, J. and Stokey, N. (1981) "The Value of Information in the Delegation Problem", Discussion paper Harvard University

- Gibbons, R. (1988) "Learning in Equilibrium Models of Arbitration", American Economic Review 78(5), 896-912

- Holmström, B. (1984), "On the Theory of Delegation" in Boyer, M. and Kihlstrom, R. (eds.), Bayesian Models in Economic Theory (Elsevier Science Publishers B.V.)

- Holmström, B. (1978) "On Incentives and Control in Organizations" Ph.D. Dissertation, Stanford University

- Holmström, B. (1979) "Moral Hazard and Observability" Bell Journal of Economics 10, $74-91$

- Holmström, B. and Ricart i Costa, J. (1986) "Managerial Incentives and Capital Management" Quarterly Journal of Economics, 835-860

- Kessler, A. (1998) "The Value of Ignorance", Rand Journal of Economics, 339-354

- Laffont, J.-J., and Martimort, D. (2002) "The Principal-Agent Model" Princeton University Press, Princeton and Oxford 
- Laux (2001) "Delegated Information Acquisition and Capital Budgeting: On the Separation of Project Evaluation and Management" Journal of Institutional and Theoretical Economics $157(4)$,

- Lewis, T. and Sappington, D. (1993) "Ignorance in Agency Problems" Journal of Economic Theory 61, 169-183

- Lewis, T. and Sappington, D. (1997) "Information Management in Incentive Problems" Journal of Political Economy 105(4), 796-821

- Li, H. (2001) "A Theory of Conservatism" Journal of Political Economy 109(3), 617-636

- Melumad, N. and Shibano, T. (1991) "Communication in Settings with no Transfers" Rand Journal of Economics 22(2), 173-198

- Milgrom, P. and Roberts, J. (1994) "Comparing Equilibria" American Economic Review 84(3), $441-459$

- Osborne K. (1989) "Optimal Forecasting Incentives", Journal of Political Economy 97(5), 1091-1112

- Ottaviani, M. and Sorensen, P. (2000) "Professional Advice" mimeo UCL

- Prendergast, C. (1993) "A Theory of "Yes Man" American Economic Review, 83(4), 757-770

- Prendergast, C. and Stole, L. (1996) "Impetuous Youngsters and Jaded Old-Timers: Acquiring a Reputation for Learning" Journal of Political Economy 104(6), 1005-1134

- Stoughton, N. (1993) "Moral Hazard and the Portfolio Management Problem" Journal of Finance 48(5), 2009-2028

- Szalay, D. (2000) "Optimal Delegation", mimeo University of Mannheim

- Szalay, D. (2001) "On the One-handed Economist", mimeo University of Mannheim

- Szalay, D. (2003) "On information acquisition in delegated portfolio management", mimeo University of Lausanne

- Tirole, J. (1999) "Incomplete Contracts: Where Do We Stand?" Econometrica 67(4), 741-781 